OPTICAL AND DYNAMIC PROPERTIES

OF UNDOPED AND DOPED

SEMICONDUCTOR

NANOSTRUCTURES

C. D. Grant, J. Z. Zhang

October 17, 2007

Annual Review of Nano Research 
This document was prepared as an account of work sponsored by an agency of the United States government. Neither the United States government nor Lawrence Livermore National Security, LLC, nor any of their employees makes any warranty, expressed or implied, or assumes any legal liability or responsibility for the accuracy, completeness, or usefulness of any information, apparatus, product, or process disclosed, or represents that its use would not infringe privately owned rights. Reference herein to any specific commercial product, process, or service by trade name, trademark, manufacturer, or otherwise does not necessarily constitute or imply its endorsement, recommendation, or favoring by the United States government or Lawrence Livermore National Security, LLC. The views and opinions of authors expressed herein do not necessarily state or reflect those of the United States government or Lawrence Livermore National Security, LLC, and shall not be used for advertising or product endorsement purposes. 


\title{
CHAPTER
}

\section{OPTICAL AND DYNAMIC PROPERTIES OF UNDOPED AND DOPED SEMICONDUCTOR NANOSTRUCTURES}

\author{
Jin Z. Zhang ${ }^{1 *}$ and Christian D. Grant ${ }^{2}$ \\ ${ }^{I}$ Department of Chemistry and Biochemistry, University of California, Santa Cruz, \\ CA 95064 USA \\ ${ }^{2}$ Lawrence Livermore National Laboratories, Livermore, CA 94550 USA \\ *Corresponding author, email: zhang@chemistry.ucsc.edu
}

This chapter provides an overview of some recent research activities on the study of optical and dynamic properties of semiconductor nanomaterials. The emphasis is on unique aspects of these properties in nanostructures as compared to bulk materials. Linear, including absorption and luminescence, and nonlinear optical as well as dynamic properties of semiconductor nanoparticles are discussed with focus on their dependence on particle size, shape, and surface characteristics. Both doped and undoped semiconductor nanomaterials are highlighted and contrasted to illustrate the use of doping to effectively alter and probe nanomaterial properties. Some emerging applications of optical nanomaterials are discussed towards the end of the chapter, including solar energy conversion, optical sensing of chemicals and biochemicals, solid state lighting, photocatalysis, and photoelectrochemistry. 


\section{Introduction}

Nanomaterials are the cornerstones of nanoscience and nanotechnology and are anticipated to play an important role in future economy, technology, and human life in general. The strong interests in nanomaterials stem from their unique physical and chemical properties and functionalities that often differ significantly from their corresponding bulk counterparts. Many of these unique properties are extremely promising for emerging technological applications, including nanoelectronics, nanophotonics, biomedicine, information storage, communication, energy conversion, catalysis, environmental protection, and space exploration.

One of the most fascinating and useful aspects of nanomaterials is their optical properties, including linear and non-linear absorption, photoluminescence, electroluminescence, and light scattering. For instance, semiconductor nanomaterials with spatial features on the order of a few nanometers exhibit dramatic size dependence of optical properties due to the quantum confinement effect $[1,2]$. Shape and interaction between particles can also play an important role [3-5]. Therefore, their optical properties can be varied for different applications by controlling the size and shape of the nanostructures.

Since the surface-to-volume ratio $(1 / \mathrm{R}$ scaling for spherical nanoparticles with radius R) is exceedingly large for nanomaterials, typically a million-fold increase compared to bulk, many of their properties, including optical, are extremely sensitive to surface characteristics [6]. As a result, one could also manipulate or modify the surface to influence and control their properties. Understanding of the surface properties of nanomaterials at the atomic level is still quite primitive at the present time.

While static studies, e.g. microscopy and XRD, provide important information about crystalline structure, size, shape, and surface, dynamic studies of charge carriers can provide complementary information that cannot be easily obtained from steady-state or time-integrated studies [7]. For example, the lifetime of charge carriers and their corresponding relaxation pathways determined from time-resolved studies can help gain insight into the effects of bandgap trap states that are due to surface or internal defects.

In the rest of this article, we will provide an overview of some recent research activities in the study of optical and dynamic properties of semiconductor nanomaterials. We will briefly discuss synthesis and structural characterization in order to make the article more self-contained. While we draw specific examples mostly from our own work, we attempt to cover as much relevant work as possible within the limited space. Even though we try our best to provide a balanced presentation of all the work cited, the viewpoints expressed in this article clearly reflects primarily our own interpretation and understanding. 


\section{Synthesis of Semiconductor Nanomaterials}

Semiconductor nanostructures are synthesized by either chemical or physical methods. In a typical chemical synthesis, reactants are mixed in an appropriate solvent to produce the nanostructured product of interest. The result of the synthesis depends strongly on a number of factors such as concentration, temperature, mixing rate, or $\mathrm{pH}$ if in aqueous solution [8-15]. Surfactant or capping molecules are often used to stabilize the nanoparticles and can even direct particle growth along a particular crystal plane into that of a rod or other structure [16]. Truly bare or naked nanoparticles are not thermodynamically stable because of high surface tension and dangling chemical bonds on the surface. Impurities either from starting materials or introduced from some other source during synthesis can have deleterious effects by either profoundly altering their optical, structural, or chemical properties or even preventing the formation of the desired nanostructure. In light of this, extreme care should be taken to ensure that high purity reactants are used and that synthetic technique is as clean as possible.

Physical methods usually involve deposition onto appropriate substrate of the desired material from a source that is evaporated by heat or other type of energy such as light. Most techniques of nanomaterials synthesis are a combination of chemical and physical methods, such as CVD (chemical vapor deposition) or MOCVD (metal organic chemical vapor deposition) [17-25]. In CVD, a precursor, often diluted in a carrier gas or gasses, is delivered into a reaction chamber at approximately ambient temperatures. As it passes over or comes into contact with a heated substrate, it reacts or decomposes to form a solid phase that is deposited onto the substrate. The substrate temperature is critical and can influence what reaction takes place. The crystal structure of the substrate surface, along with other experimental parameters, determines what nanostructures can be generated. In MOCVD, atoms to be incorporated in a crystal of interest are combined with complex organic gas molecules and passed over a hot semiconductor wafer. The heat decomposes the molecules and deposits the desired atoms onto the substrate's surface. By controlling the composition of the gas, one can vary the properties of the crystal on an atomic scale. The crystal structure of the fabricated materials is dictated by the crystal structure of the substrate.

A special technique for synthesizing nanostructures, especially 1-D structures such as nanowires, is based on the VLS (vapor-liquid-solid) mechanism first discovered in the mid-1960s [26-29]. The mechanism consists of small metal particle catalysts deposited on a substrate. The substrate is then heated and vapor (e.g. $\mathrm{Si}, \mathrm{ZnO}, \mathrm{GaN}$ ) of the material of choice is introduced. The vapor diffuses into the metal until a saturated solution is generated and the material of choice precipitates forming nanowires. There are several modern examples using VLS to grow many different types of nanowires or other one-dimensional nanostructures [30- 
34]. One such variation is where a laser ablates a substrate containing a metal/semiconductor mixture to create a semiconductor/metal molten alloy [35-37]. The resulting nanowires undergo VLS growth. Nanowires made by the laser assisted catalytic growth have lengths up to several $\mu \mathrm{m}$ [38-41].

The above synthetic techniques are generally considered bottom-up approaches where atoms and molecules are brought together to produce larger nanostructures. An opposite approach is top-down where large bulk scale structures are fabricated into smaller nanostructures. Lithographic techniques such as E-beam or photo-lithography are examples that allow creation of nanostructures on the micron and nanometer scales, easily down to tens of nanometers [42]. Such techniques lend conveniently for mass production of high quality and high purity structures critical in microelectronics and computer industry. It is currently a challenge to create structures on a few $\mathrm{nm}$ scale using typical lithographic methods. There is urgent need for developing new technologies that can meet this challenge, especially for the microelectronics and computer industry. The combination of top-down and bottom-up approaches may hold the key to solving this problem in the future.

There are a number of review articles and books that devote a significant amount of detail on nanomaterial synthesis [3, 5, 43-46], and we refer the reader to these resources since this chapter focuses more on the optical properties of semiconductor nanomaterials and their applications.

\section{Structural Characterization}

Structural determination and understanding are an important and integral part of nanomaterials research. Since the nanostructures are too small to be visualized with a conventional optical microscope, it is essential to use appropriate tools to characterize their structure in detail at the molecular or atomic level. This is important not only for understanding their fundamental properties but also for exploring their functional and technical performance in technological applications. There are a number of powerful experimental techniques that can be used to characterize structural and surface properties of nanomaterials either directly or indirectly, e.g. XRD (X-ray diffraction), STM (scanning tunneling microscopy), AFM (atomic force microscopy), SEM (scanning electron microscopy), TEM (transmission electron microscopy), XAS (X-ray absorption spectroscopy) such as EXAFS (extended X-ray absorption fine structure) and EXANES(extended X-ray absorption near edge structure), EDX (energy dispersive X-ray), XPS (X-ray photoelectron spectroscopy), IR (infrared), Raman, and DLS (dynamic light scattering) [43, 44, 47-49]. Some of these techniques are more surface sensitive than others.. Some of the techniques are directly element-specific while others are not. The choice of technique depends strongly on the information being sought about the material. 
$\mathrm{X}$-ray diffraction (XRD) is a popular and powerful technique for determining crystal structure of crystalline materials. Diffraction patterns at wide-angles are directly related to the atomic structure of the nanocrystals, while the pattern in the small-angle region yields information about the ordered assembly of nanocrystals, e.g. superlattices $[3,50,51]$. By examining the diffraction pattern, one can identify the crystalline phase of the material. Small angle scattering is useful for evaluating the average interparticle distance while wide-angle diffraction is useful for refining the atomic structure of nanoclusters Alivisatos, 1996, 933]. The widths of the diffraction lines are closely related to the size, size distribution, defects, and strain in nanocrystals. As the size of the nanocrystal decreases, the line width is broadened due to loss of long range order relative to the bulk. This XRD line width can be used to estimate the size of the particle by using the Debye-Scherrer formula. However, this line broadening results in inaccuracies in the quantitative structural analysis of nanocrystals smaller than $\sim 1 \mathrm{~nm}$.

Scanning probe microscopy (SPM) represents a group of techniques, including scanning tunneling microscopy (STM), atomic force microscopy (AFM), and chemical force microscopy, that have been extensively applied to characterize nanostructures $[47,52]$. A common characteristic of these techniques is that an atom sharp tip scans across the specimen surface and the images are formed by either measuring the current flowing through the tip or the force acting on the tip. SPM can be operated in a variety of environmental conditions, in a variety of different liquids or gases, allowing direct imaging of inorganic surfaces and organic molecules. It allows viewing and manipulation of objects on the nanoscale and its invention is a major milestone in nanotechnology.

STM is based on the quantum tunneling effect [53]. The wave function of the electrons in a solid extends into the vacuum and decay exponentially. If a tip is brought sufficiently close to the solid surface, the overlap of the electron wave functions of the tip with that of the solid results in the tunneling of the electrons from the solid to the tip when a small electric voltage is applied. Images are obtained by detecting the tunneling current when the bias voltage is fixed while the tip is scanned across the surface, because the magnitude of the tunneling current is very sensitive to the gap distance between the tip and the surface. Based on current-voltage curves measured experimentally, the surface electronic structure can also be derived. Therefore, STM is both an imaging as well as a spectroscopy technique. STM works primarily for conductive specimens or for samples on conducting substrates.

For non-conductive nanomaterials, atomic force microscopy (AFM) is a better choice [52, 54]. AFM operates in an analogous mechanism except the signal is the force between the tip and the solid surface. The interaction between two atoms is repulsive at short-range and attractive at long-range. The force acting on the tip reflects the distance from the tip atom(s) to the surface atom, thus images can be formed by detecting the force while the 
tip is scanned across the specimen. A more generalized application of AFM is scanning force microscopy, which can measure magnetic, electrostatic, frictional, or molecular interaction forces allowing for nanomechanical measurements.

Scanning electron microscopy (SEM) is a powerful and popular technique for imaging the surfaces of almost any material with a resolution down to about $1 \mathrm{~nm}[48,49]$. The image resolution offered by SEM depends not only on the property of the electron probe, but also on the interaction of the electron probe with the specimen. Interaction of an incident electron beam with the specimen produces secondary electrons, with energies typically smaller than $50 \mathrm{eV}$, the emission efficiency of which sensitively depends on surface geometry, surface chemical characteristics and bulk chemical composition [55].

Transmission electron microscopy (TEM) is a high spatial resolution structural and chemical characterization tool.[56] A modern TEM has the capability to directly image atoms in crystalline specimens at resolutions close to $0.1 \mathrm{~nm}$, smaller than interatomic distance. An electron beam can also be focused to a diameter smaller than $\sim 0.3 \mathrm{~nm}$, allowing quantitative chemical analysis from a single nanocrystal. This type of analysis is extremely important for characterizing materials at a length scale from atoms to hundreds of nanometers. TEM can be used to characterize nanomaterials to gain information about particle size, shape, crystallinity, and interparticle interaction [48, 57].

$\mathrm{X}$-ray based spectroscopies are useful in determining the chemical composition of materials. These techniques include X-ray absorption spectroscopy (XAS) such as extended X-ray absorption fine structure (EXAFS) and X-ray absorption near edge structure (XANES), X-ray Fluorescence spectroscopy (XRF), energy dispersive X-ray spectroscopy (EDX), and X-ray photoelectron spectroscopy (XPS) [58, 59]. They are mostly based on detecting and analyzing radiation absorbed or emitted from a sample after excitation with X-rays, with the exception that electrons are analyzed in XPS. The spectroscopic features are characteristic of specific elements and thereby can be used for sample elemental analysis. This is fundamentally because each element of the periodic table has a unique electronic structure and, thus, a unique response to electromagnetic radiation such as X-rays.

XAS is an element-specific probe of the local structure of atoms or ions in a sample. Interpretation of XAS spectra commonly uses standards with known structures, but can also be accomplished using theory to derive material structure. In either case, the species of the material is determined based on its unique local structure. X-ray absorption spectroscopy results form the absorption of a high energy X-ray by an atom in a sample. This absorption occurs at a defined energy corresponding to the binding energy of the electron in the material. The ejected electron interacts with the surrounding atoms to produce the spectrum that is observed. Occasionally, the electron can be excited into vacant bound electronic states near the 
valence or conduction bands. As a result, distinct absorptions will result at these energies. Often these features are diagnostic of coordination. XAS is commonly divided into two spectral region. The first is the X-ray absorption near edge structure or the XANES spectral region [59]. The XANES technique is sensitive to the valence state and speciation of the element of interest, and consequently is often used as a method to determine oxidation state and coordination environment of materials. XANES spectra are commonly compared to standards to determine which species are present in an unknown sample. XANES is sensitive to bonding environment as well as oxidation state and thereby it is capable of discriminating species of similar formal oxidation state but different coordination. The high energy region relative to XANES of the X-ray absorption spectrum is termed the extended X-ray absorption fine structure or EXAFS region. EXAFS yields a wealth of information, including the identity of neighboring atoms, their distance from the excited atom, the number of atoms in nearest neighbor shell, and the degree of disorder in the particular atomic shell. These distances and coordination numbers are diagnostic of a specific mineral or adsorbate-mineral interaction; consequently, the data are useful to identify and quantify major crystal phases, adsorption complexes, and crystallinity.

$\mathrm{X}$-ray fluorescence (XRF) is a technique used to determine elemental composition in a material. The technique is based on irradiating a sample with either a lab based X-ray source (X-ray tube) or monochromatic radiation such as that obtained from a synchrotron. The emitted $\mathrm{X}$-rays are characteristic of the element contained in the material. In contrast, energy dispersive X-ray spectroscopy (EDS or EDX) is usually based on direct sample excitation with an electron beam (as in an SEM) with subsequent detection of an emitted X-ray. In either case, the information obtained from either XRF or EDX is equivalent in that it is chemically specific.

XPS is based on the measurement of photoelectrons following X-ray excitation of a sample. It is a quantitative spectroscopic technique that measures the chemical composition, redox state, and electronic state of the elements within a material. XPS spectra are obtained by irradiating a material with a beam of X-rays while simultaneously measuring the kinetic energy and number of electrons that escape from the top 1 to $10 \mathrm{~nm}$ of the material being analyzed. Thus, XPS is a surface sensitive analytic technique and it requires ultra high vacuum (UHV) conditions [58].

Optical spectroscopy such as IR and Raman provide more direct structure information while UV-visible electronic absorption and photoluminescence (PL) provide indirect structural information. For example, higher crystallinity and large particle size result in sharper Raman peaks and strong Raman signal. Disorder or high density of defects are reflected in low PL yield and trap state emission [7, 43]. Dynamic light scattering (DLS) can provide a measure of the overall size of nanoparticles in solution, usually when the size is larger than a few $\mathrm{nm}$. In general 
optical spectroscopy is sensitive to structural properties but cannot provide a direct probe of the structural details.

\section{Optical Properties}

Semiconductor nanoparticles or quantum dots (QDs) have rich optical properties that strongly depend on size, especially when the particle size is less than the exciton Bohr radius of the material. Exciton Bohr radii are typically on the order of a few $\mathrm{nm}$ for semiconductors like CdSe, but are smaller for metal oxides like $\mathrm{TiO}_{2}$. Their optical properties are also very sensitive to the surface characteristics and, to a lesser degree, of shape of the nanoparticles. For example, the photoluminescence spectrum and quantum yield can be altered by orders of magnitude by surface modification of the nanoparticles [60]. This fact can be used to advantage for specific applications of interest. Another factor affecting the optical properties is the interaction between nanoparticles or between the nanoparticles and their embedding environment [3,4]. Interaction between nanoparticles typically leads to lower PL quantum yield and red-shifted PL spectrum due to shortened charge carrier lifetime [61]. Interaction with the environment is more complex and depends strongly on the chemical and physical nature of environment medium.

Optical properties are commonly characterized using spectroscopic techniques including UV-visible and photoluminescence spectroscopy, which both yield information about the electronic structure of nanoparticles. Related optical techniques such as Raman and IR provide information about the crystal structure such as phonon or vibrational frequencies and crystal phases. There are also a number of other more specialized optical techniques, often laser-based, that have been used to characterize the linear or non-linear optical properties of nanomaterials, such as second harmonic generation (SHG) [62-64], sum-frequency generation (SFG) [65, 66], and four-wave mixing [67].

\subsection{Linear Optical Absorption and Emission}

A striking optical signature of nanoparticles or quantum dots (QDs) is the strong size dependence of the absorption and photoluminescence (PL) (Figure 1 right) especially when the particle size is comparable to the exciton Bohr radius. An experimental manifestation of the size dependence is the blue shift of the UV-visible and PL spectra with decreasing particle size. This behavior is due to what is termed quantum confinement. The quantum confinement effect may be qualitatively understood using the particle-in-a-box model from quantum mechanics. In other words, a smaller box yields larger energy gaps between electronic states than does a larger box. For spherical particles, a quantification of quantum confinement is embodied in equation $1[1,2]$, 


$$
E_{g, \text { effective }}(R)=E_{g}(\infty)+\frac{\hbar^{2} \pi^{2}}{2 R^{2}}\left(\frac{1}{m_{e}}+\frac{1}{m_{h}}\right)-\frac{1.8 e^{2}}{\varepsilon R},
$$

where $\mathrm{E}_{\mathrm{g}}(\infty)$ is the bulk bandgap, $\mathrm{m}_{\mathrm{e}}$ and $\mathrm{m}_{\mathrm{h}}$ are the effective masses of the electron and hole, and $\varepsilon$ is the bulk optical dielectric constant or relative permittivity. The second term on the right hand side shows that the effective bandgap is inversely proportional to $\mathrm{R}^{2}$ and increases as size decreases. On the other hand, the third term shows that the bandgap energy decreases with decreasing $\mathrm{R}$ due to increased Columbic interaction. However, since the second term becomes dominant with small $\mathrm{R}$, the effective bandgap is expected to increase with decreasing $R$, especially when $\mathrm{R}$ is small. This effect is illustrated schematically in Figure 1 (left). The effect of solvent or embedding environment is neglected in this form of the equation, but the effect of solvation is typically small compared to quantum confinement.

The quantum size confinement effect becomes significant particularly when the particle size becomes comparable to or smaller than the Bohr exciton radius, $\alpha_{B}$, which is given by:

$$
\alpha_{\mathrm{B}}=\frac{\varepsilon_{0} \varepsilon \mathrm{h}^{2}}{\pi \mu \mathrm{e}^{2}}
$$

where $\varepsilon_{0}$ and $\varepsilon$ are the permittivity of vacuum and relative permittivity of the semiconductor, $\mu$ is the reduced mass of the electron and hole, $\mathrm{m}_{\mathrm{e}} \mathrm{m}_{\mathrm{h}} /\left(\mathrm{m}_{\mathrm{e}}+\mathrm{m}_{\mathrm{h}}\right)$, and e the electron charge. For instance, the Bohr radius of $\mathrm{CdS}$ is around $2.4 \mathrm{~nm} \mathrm{[68]} \mathrm{and} \mathrm{particles} \mathrm{with} \mathrm{radius} \mathrm{smaller} \mathrm{or} \mathrm{comparable}$ to $2.4 \mathrm{~nm}$ show strong quantum confinement effects, as indicated by a significant blue-shift of their optical absorption relative to that of bulk [6971]. Likewise, the absorption spectra of CdSe nanoparticles (NPs) show a dramatic blue-shift with decreasing particle size [72]. The emission spectra usually show a similar blue shift with decreasing size. Figure 1 (right) displays different sized CdTe nanoparticles exhibiting different PL center wavelengths with larger particles (left) showing redder luminescence.
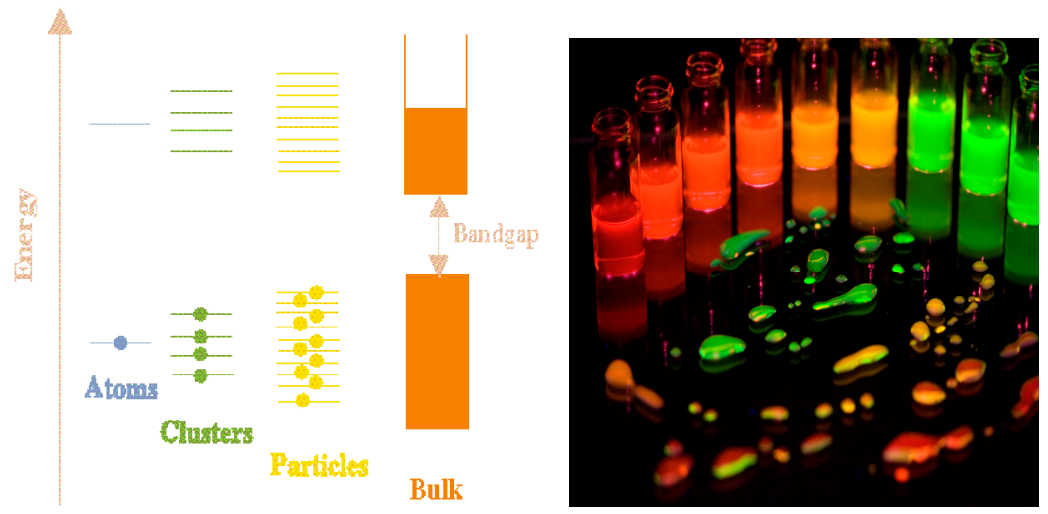

Figure 1. (left) Illustration of quantum confinement effect in different systems ranging from atoms to bulk materials. (Right) Photos of CdTe QDs with different sizes under UV illumination, ranging from $6 \mathrm{~nm}$ (red) to $2.5 \mathrm{~nm}$ (green) in size [73]. 
The UV-visible absorption measured as a function of wavelength reflects the strength of the electronic transition between the valence (VB) and conduction bands (CB). The transition from the valence to the conduction band is the solid state analog to the HOMO-LUMO electronic transition in molecules. In the case of direct bandgap transitions, typically a strong excitonic band with a well-defined peak is observed at the low energy side of the spectrum. The excitonic state is located slightly below the bottom of the conduction band. The energy difference between the bottom of the $\mathrm{CB}$ and the excitonic state is the electron-hole binding energy, which is typically a few to a few hundred $\mathrm{meV}$. Thus, the peak position of the excitonic absorption band provides an estimate of the bandgap of the nanoparticle. The bandgap energy increases with decreasing particle size, resulting in a blue-shift of the absorption spectrum as well as the excitonic peak. In contrast, indirect bandgap transitions lack an excitonic peak and the spectrum usually features a gradually and smoothly increasing absorption with decreasing wavelength. A wellknown example is Si $[7,74]$. Quantum confinement in indirect bandgap materials is less easily observable due to the lack of sharp or well-defined spectral peaks or bands. The intensity of the absorbance for QDs follows Beer's law. In this case, QDs can be considered as large molecules. Each QD typically contains a few hundred to a few thousands atoms and the absorption oscillator strength for one QD is proportional to the number of atoms in each QD [75, 76]. An experimental study by $\mathrm{Yu}$ et al. determining the molar absorptivity of $\mathrm{CdS}, \mathrm{CdSe}$, and CdTe as a function of size bears this out quite well [77].

In PL spectroscopy, photoemission is measured following excitation of the sample with a fixed wavelength of light. Photoluminescence reflects the electronic transition from the excited state, usually the excitonic state but also could be trap states, to the ground state, the valence band. Since PL is a "zero-background" experiment, it is much more sensitive, by approximately 1000 times, than UV-visible absorption measurements [78]. Thus PL provides a sensitive probe of bandgap states that UV-visible spectroscopy is much less sensitive to. For a typical nanoparticle sample, PL can be generally divided into bandedge emission, including excitonic emission, and trap state emission. If the size distribution is very narrow, bandedge luminescence is often characterized by a small Stokes shift from the excitonic absorption band along with a narrow bandwidth which usually means there is a narrow energy distribution of emitting states. In contrast, trap states are typically located within the semiconductor bandgap and hence their emission is usually red shifted relative to bandedge emission. In addition, trap state PL is often characterized by a large bandwidth reflecting a broad energy distribution of emitting states. The ratio between the two types of emission is determined by the density and distribution of trap states. Strong trap state emission indicates a high density of trap states and efficient electron and/or hole trapping. 
It is possible to prepare high quality samples that have mostly bandedge emission when the surface is well passivated. For example, TOPO (tri- $n$-octylphosphine oxide) capped CdSe show mostly bandedge emission and weak trap state emission, which is an indication of a high quality sample $[8,79,80]$. Luminescence can also be enhanced by surface modification [81-86] or using core/shell structures [12, 87-89]. Many nanoparticles, including $\mathrm{CdSe}, \mathrm{CdS}, \mathrm{ZnS}$, have been found to show strong photoluminescence [90]. Other nanoparticles have generally been found to be weakly luminescent or non-luminescent at room temperature, e.g. $\mathrm{PbS}$ [91], $\mathrm{PbI}_{2}$ [92], $\mathrm{CuS}$ [93], $\mathrm{Ag}_{2} \mathrm{~S}$ [94]. The low luminescence can be due to either the indirect nature of the semiconductor or a high density of internal and/or surface trap states that quench the luminescence. Luminescence usually increases at lower temperature due to suppression of electronphonon interactions and thereby increases the excited electronic state lifetime. Controlling the surface by removing surface trap states can lead to significant enhancement of luminescence as well as of the ratio of bandedge over trap state emission [81-86]. Surface modification often involves capping the particle surface with organic, inorganic, biological molecules, or even ions that reduce the amount of trap states that quench luminescence. This scheme likely removes surface trap states, enhances luminescence, and is important for many applications that require highly luminescent nanoparticles, e.g. lasers, LEDs, fluorescence imaging, and optical sensing.

One common issue encountered is PL quenching in solution over time. The reason for quenching varies and may be influenced by such factors as $\mathrm{pH}$, the presence of $\mathrm{O}_{2}, \mathrm{CO}$, or other gas molecules, or even room light [95, 96]. More specifically, $\mathrm{pH}$ is one critical factor to consider if the particles need to be in aqueous solution. There is indirect evidence that acidic conditions may result in dissolution of the oxide or hydroxide layer present on the surface of the nanoparticle that serves to stabilize the QD's luminescence. When the protecting layer is dissolved under acidic conditions, there is an increase in surface trap or defect states that quench the PL [60]. Whatever the true reason for the PL quenching, the luminescence intensity decay over time presents a problem for applications like biological labeling or imaging. To address the problem, different approaches have been considered and used, primarily in terms of stabilizing the surface by using a protecting layer of another material, e.g. polymer, large bandgap semiconductor like $\mathrm{ZnS}$, or insulator such as silica and polymers [97, 98]. One interesting example is $\mathrm{SiO}_{2}$ coated CdTe nanoparticles [73]. As shown in Figure 2, the PL of CdTe QDs lacking a silica coating is quenched within $200 \mathrm{~s}$ when dispersed in a tris-borate EDTA (TBE) buffer solution (blue curve). TBE is a commonly used buffer in molecular biology involving nucleic acids, so determining the PL stability of QDs in this relevant buffer is important for biological applications. It should be pointed out that in $\mathrm{SiO}_{2}$ coated CdTe the PL intensity will not decay or decreases only slightly if they are dissolved in 
water. With only a partial layer of silica coating, the PL is better stabilized (red curve) and the intensity lasts slightly longer than uncoated CdTe (blue curve) in TBE buffer. However, when a 2-5 nm shell of silica coats the CdTe QD surface, the PL (green curve) persists longer than the uncoated or only partially coated CdTe. Attempts have been made recently in our lab to put even thicker layers of silica with the hope that the PL will be more stable for even longer. PL stability in biologically relevant buffers is essential for many PL based applications such as biomarker detection [73].

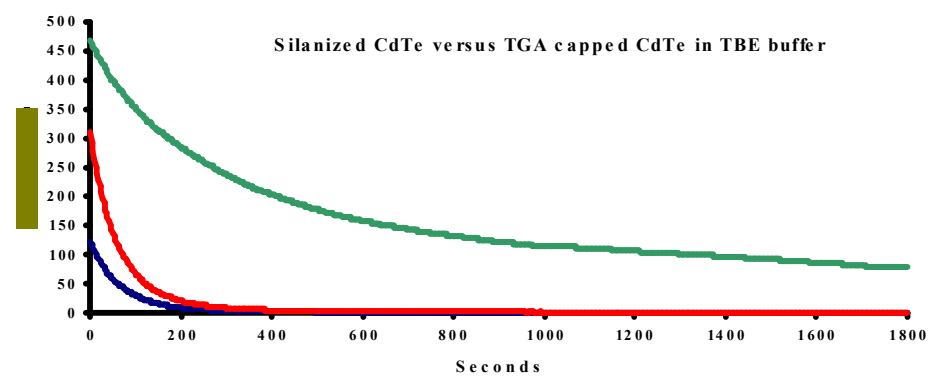

Figure 2. Effect of silica coating on the PL intensity in TBE buffer of CdTe nanoparticles. Adapted with permission from ref. [73]. Uncoated particles are shown in blue, partial silica coated in red, and with a 2-5 nm silica shell in green.

In addition to PL emission spectroscopy, another very useful PL experimental technique is photoluminescence excitation (PLE). This involves varying the wavelength of excitation while monitoring the PL intensity at a fixed wavelength. In the simplest case of a single emitting species (or state), the PLE is identical to the absorption. However, when there are several species present (e.g. different sized QDs) or a single species that exists in different forms in the ground state, the PLE and absorption bands are no longer superimposable. This technique can yield information about the nature of the emitting state or species. Specifically, in the case of ZnSe:Mn or ZnS:Mn QDs by monitoring the emission from the Mn dopant, the PLE band is identical to the absorption band indicating that the emission from $\mathrm{Mn}$ is due to energy transfer through excitation of the host crystal. Comparison of absorption of PLE often provides useful information on the types of states that are contributing to the PL.

There are two practical problems that are often encountered in PL measurements: Raman scattering and high order Rayleigh scattering. Raman scattering from solvent molecules can show up as relatively strong signal in PL spectroscopy, especially when the PL intensity is low. For nanoparticles, PL speaks are generally broad for ensemble samples while Raman peaks are usually narrow. A simple diagnostic to verify that a peak is due to Raman scattering is by changing the excitation wavelength and observe if the peak shifts accordingly. If the observed peak is from Raman scattering, it will shift by the same amount in frequency as the change in 
excitation wavelength, while there will be no shift if the emission is due to true PL.

Another potential artifact is high order Rayleigh scattering that occur at multiples of the excitation wavelength, $\lambda$. For example, if $\lambda=400 \mathrm{~nm}$ is the excitation wavelength, due to the basic grating diffraction equation, $10^{-6} n k \lambda=\sin \alpha+\sin \beta$ (where $n$ is the groove density of the grating, $k$ is the diffraction order, $\alpha$ the angle of incidence, and $\beta$ is the angle of diffraction), apparent "peaks" at $n \times 400 \mathrm{~nm}$ can show up on the PL spectrum, e.g. 400 $\mathrm{nm}, 800 \mathrm{~nm}$ and $1200 \mathrm{~nm}$ corresponding to $k=1,2$, and 3 respectively, if the spectrometer scans cover these regions. Such apparent peaks do not correspond to real light at these wavelengths but are simply a grating effect from the $400 \mathrm{~nm}$ Rayleigh scattering. One indication is their narrow line widths. To determine this experimentally, one can use short or long pass optical filters to check if the observed peaks are from the sample or artifact from the instrument. For instance, if a peak at $800 \mathrm{~nm}$ does not disappear when a filter that blocks $800 \mathrm{~nm}$ light is placed in front of the detector, it is most likely that this peak is a second order Rayleigh scattering from the $400 \mathrm{~nm}$ excitation light. Of course, the first order $400 \mathrm{~nm}$ is usually blocked by a filter. But there is usually still $400 \mathrm{~nm}$ light leaking through the filter. Usually it is a good idea to try to avoid observing the first order excitation light directly by starting the PL spectral scan to the red of the excitation line. Of course the choice of PL scan range depends on the emission properties of the nanomaterial under consideration. Confounding mistakes of this sort due to Raman and Rayleigh scattering have appeared in the literature more often than expected.

\subsection{Non-linear Optical Absorption and Emission}

Similar to bulk materials, nanomaterials exhibit non-linear optical properties such as multiphoton absorption or emission, harmonic generation, up- or down-conversion. Nanoparticles have interesting nonlinear optical properties at high excitation intensities, including absorption saturation, shift of transient bleach, third and second harmonic generation, and up-conversion luminescence. The most commonly observed nonlinear effect in semiconductor nanoparticles is absorption saturation and transient bleach shift at high intensities [82, 86, 99-104]. Similar nonlinear absorption have been observed for quantum wires of GaAs [105, 106] and porous Si $[107,108]$. These non-linear optical properties have been considered potentially useful for optical limiting and switching applications [109].

Another non-linear optical phenomenon is harmonic generation, mostly based on the third-order nonlinear optical properties of semiconductor nanoparticles [110-113]. The third order non-linearity is also responsible for phenomena such as the Kerr effect and degenerate four wave mixing (DFWM) [114]. For instance, the third order non-linear susceptibility, $\chi^{(3)}\left(\sim 5.6 \times 10^{-12} \mathrm{esu}\right)$ for $\mathrm{PbS}$ nanoparticles has been 
determined using time-resolved optical Kerr effect spectroscopy and it was found to be dependent on surface modification [113]. Third order nonlinearity of porous silicon has been measured with the Z-scan technique and found to be significantly enhanced over crystalline silicon [109]. DFWM studies of thin films containing CdS nanoparticles found a large $\chi^{(3)}$ value, $\sim 10^{-7} \mathrm{esu}$, around the excitonic resonance at room temperature [115].

Only a few studies have been carried out on second-order nonlinear optical properties since it is usually believed that the centrosymmetry or near centrosymmetry of the spherical nanoparticles reduces their firs-order hyperpolarizability $(\beta)$ to zero or near zero. Using hyper-Rayleigh scattering, second harmonic generation in CdSe nanocrystals has been observed [116]. The first hyperpolarizability $\beta$ per nanocrystal was found to be dependent on particle size, decreasing with size down to about $1.3 \mathrm{~nm}$ in radius and then increasing with further size reduction. These results are explained in terms of surface and bulk-like contributions. Similar technique has been used for CdS nanoparticles for which the $\beta$-value per particle ( $4 \mathrm{~nm}$ mean diameter) was found to be on the order of $10^{-27}$ esu, which is quite high for solution species [117]. Second harmonic generation has also been observed for magnetic cobalt ferrite $\left(\mathrm{CoFe}_{2} \mathrm{O}_{4}\right)$ colloidal particles when oriented with a magnetic field [118]. The nonlinear optical properties of nanoparticles are found to be strongly influenced by the surface.

As discussed earlier, the optical properties of isolated nanoparticles can be very different from those of assembled nanoparticle films. This is true for both linear and non-linear optical properties. Theoretical calculations on nonlinear optical properties of nanoparticle superlattice solids have shown that an ideal resonant state for a nonlinear optical process is the one that has large volume and narrow line width [119-121]. The calculations also showed that nonlinear optical responses could be enhanced greatly with a decrease in interparticle separation distance.

Anti-Stokes photoluminescence or photoluminescence up-conversion is another interesting non-linear optical phenomenon. In contrast to Stokes emission, the photon energy of the luminescence output is higher than the excitation photon energy. This effect has been previously reported for both doped [122, 123] and high purity bulk semiconductors [124, 125]. For bulk semiconductors, the energy up-conversion is usually achieved by (i) an Auger recombination process, (ii) anti-Stokes Raman scattering mediated by thermally populated phonons, or (iii) two-photon absorption [126, 127]. Luminescence up-conversion has been observed in semiconductor heterojunctions and quantum wells [127-143] and has been explained based on either Auger recombination [131, 136, 144] or twophoton absorption [137]. Long-lived intermediate states have been suggested to be essential for luminescence up-conversion in some heterostructures such as $\mathrm{GaAs} / \mathrm{Al}_{\mathrm{x}} \mathrm{Ga}_{1-\mathrm{x}} \mathrm{As}$ [136]. For semiconductor nanoparticles or quantum dots with confinement in three dimensions, luminescence up-conversion has only recently been reported for CdS [145], 
InP [126, 146], CdSe [126], InAs/GaAs [147], and $\mathrm{Er}^{3+}$-doped $\mathrm{BaTiO}_{3}$ [148]. Surface states have been proposed to play an important role in the up-conversion in nanoparticles such as InP and CdSe [126].

Luminescence up-conversion in $\mathrm{ZnS}: \mathrm{Mn}$ nanoparticles and bulk has been observed [149]. When $767 \mathrm{~nm}$ excitation was used, $\mathrm{Mn}^{2+}$ emission near $620 \mathrm{~nm}$ was observed with intensity increasing almost quadratically with excitation intensity. The red shift of $\mathrm{Mn}^{2+}$ emission from that usually observed at $580 \mathrm{~nm}$ to $620 \mathrm{~nm}$ has been proposed to be caused by the difference in particle size. However, a more likely explanation could be the local environment of the $\mathrm{Mn}^{2+}$ ion rather than particle size. Comparison with $383.5 \mathrm{~nm}$ excitation showed similar luminescence spectrum and decay kinetics, indicating that the up-converted luminescence with $767 \mathrm{~nm}$ excitation is due to a two-photon process. The observation of fluorescence up-conversion in $\mathrm{Mn}^{2+}$-doped $\mathrm{ZnS}$ opens up some new and interesting possibilities for applications in optoelectronics, e.g. as infrared phosphors. There remain some unanswered questions, especially in terms of some intriguing temperature dependence of the up-converted luminescence [150]. It was found that the up-conversion luminescence of ZnS:Mn nanoparticles first decreases and then increases with increasing temperature. This is in contrast to bulk $\mathrm{ZnS}: \mathrm{Mn}$ in which the luminescence intensity decreases monotonically with increasing temperature due to increasing electron-phonon interaction. The increase in luminescence intensity with increasing temperature for nanoparticles was attributed tentatively to involvement of surface trap states. With increasing temperature, surface trap states can be thermally activated, resulting in increased energy transfer to the excited state of $\mathrm{Mn}^{2+}$ and thereby increased luminescence. This factor apparently is significant enough to overcome the increased electron-phonon coupling with increasing temperature that usually results in decreased luminescence [150].

Raman scattering could also perhaps be considered as a non-linear optical phenomenon since it involves two photons and inelastic scattering. Raman scattering is a powerful technique for studying molecules with specificity. For nanomaterials, Raman scattering can be used to study vibrational or phonon modes, electron-phonon coupling, as well as symmetries of excited electronic states. Raman spectra of nanoparticles have been studied in a number of cases, including CdS [151-156], CdSe [157-159] ZnS,[154] InP,[160], Si [161-164], and Ge [165-171]. Resonance Raman spectra of GaAs [172] and CdZnSe/ZnSe [173] quantum wires have also been determined. For CdS nanocrystals, resonance Raman spectrum reveals that the lowest electronic excited state is coupled strongly to the lattice and the coupling decreases with decreasing nanocrystal size [153]. Raman spectra of composite films of $\mathrm{Ge}$ and $\mathrm{ZnO}$ nanoparticles revealed a $300 \mathrm{~cm}^{-1} \mathrm{Ge}-\mathrm{Ge}$ transverse optical (TO) vibrational band of $\mathrm{Ge}$ nanocrystals, which shifted towards lower frequencies on decreasing the size of Ge nanocrystals due to phonon confinement in smaller crystallites [170]. For $4.5 \mathrm{~nm}$ nanocrystals of CdSe, 
the coupling between the lowest electronic excited state and the LO phonons is found to be 20 times weaker than in the bulk solid [157]. For $\mathrm{CdZnSe} / \mathrm{ZnSe}$ quantum wires, resonance Raman spectroscopy revealed that the $\mathrm{ZnSe}$-like LO phonon position depends on the $\mathrm{Cd}$ content as well as excitation wavelength due to relative intensity changes of the peak contributions of the wire edges and of the wire center [173].

\subsection{Other Relevant Optical Properties: Chemiluminescence and Electroluminescence}

Besides optical absorption and emission, nanomaterials have other interesting optical properties such as chemiluminescence (CL) and electroluminescence (EL) that are of interest for technological applications such as chemical sensing and biochemical detection. For example, CL has been observed for CdTe nanoparticles [174, 175]. CL in CdTe nanoparticles capped with thioglycolic acid (TGA) was induced by direct chemical oxidation in aqueous solution using hydrogen peroxide and potassium permanganate under basic conditions [175]. The oxidized CL of CdTe NCs displayed size-dependent effect and its intensity increased along with increasing the sizes of the nanoparticles. Electron and hole injection into the CdTe nanoparticles through radicals such as $\mathrm{O}_{2}^{-}$and $\mathrm{OH} \cdot$ are proposed to be responsible for the strong CL observed.

Electroluminescence has been reported in various nanoparticles including Si [176], $\mathrm{ZnO}$ [177], and $\mathrm{CdSe} / \mathrm{CdS}$ core/shells [178]. With a semiconductor polymer poly(N-vinylcarbazole) (PVK) doped with $\mathrm{CdSe} / \mathrm{CdS}$ core-shell semiconductor quantum dots (QDs), white light emission was observed and attributed to the incomplete energy and charge transfer from PVK to CdSe/CdS core-shell QDs.

\section{Charge Carrier Dynamics}

\subsection{Ultrafast Time-resolved Laser Techniques}

Study of charge carrier relaxation in nanoparticles provides complementary information to steady-state experiments that may not be readily or easily accessible using the time-integrated techniques already discussed. This charge carrier dynamical information can lead to a deeper understanding of nanomaterial fundamental properties including but not limited to optical properties. Time-resolved laser spectroscopy is a powerful technique for probing charge carrier dynamics in nanomaterials $[6,7]$. Two common techniques are transient absorption (TA) and timeresolved luminescence. In transient operation measurement, a short laser pulse excites the sample of interest, namely a pump-pulse, and a second short laser pulse (probe pulse) is used to interrogate an excited population of charge carriers, e.g. electrons. The probe pulse is delayed in time with respect to the pump or excitation pulse. Changes in the detected signal 
(transmission in the case of transient absorption) of the probe pulse with this time delay contains information of the dynamics or lifetime of the excited carriers being probed [7]. The assignment of the observed signal is usually not trivial and often control experiments are combined with other information such as theory to help make the appropriate determination of origin of the transient absorption signal. This is also partly due to the fact that the probe pulse initiates an electronic transition between two excited states that are often not well characterized, especially the higher-lying electronic state. Nonetheless, transient absorption is versatile and provides high time resolution since the instrument response is determined only by the cross correlation of the pump and probe pulses, which are usually very short temporally (easily down to a few tens of fs with current technology).

In time-resolved luminescence measurements, the excitation mechanism is the same as in transient absorption. The difference is in the monitoring of the excited population. Instead of monitoring the excited state population with a second short laser pulse, the time profile of the photoluminescence is monitored. If the PL is monitored directly with a photodetector, such as photomultiplier tube (PMT), photodiode, or chargecoupled device (CCD), the time resolution is limited by the detector, which is often much longer (ps or ns) than the excitation laser pulse. For example Time Correlated Single-Photon Counting (TCSPC) can have an instrument resolution down to $25 \mathrm{ps}$ and with instrumental deconvolution lifetimes of a few ps are reliably obtained. One way to take full advantage of short laser pulses is to use a technique called luminescence up-conversion. In this method, a second short laser pulse is mixed with the PL in a non-linear crystal to generate a new up-converted or higher energy photon which is then directed into a spectrometer and detected. The width or time-profile of the up-converted pulse is mainly determined by the second laser pulse used for the up-conversion while the energy of the photons in the upconverted pulse is the sum of the energies of photons from the PL and second up-converting laser pulse. By changing the time delay between the second laser pulse with respect to the excitation pulse, a time profile of the PL kinetics is obtained. In this case, the time resolution is much higher as it is determined by the cross overlap of the second up-converting and first pump pulse. While the PL up-conversion technique provides high time resolution for PL dynamics measurement, it is often involved and challenging since the up-converted signal is typically small due to the low PL intensity and the non-linear nature of up-conversion [179].

\subsection{Linear Dynamic Properties: Relaxation, Trapping, and Recombination}

Figure 3 provides a summary of possible dynamic processes involved in charge carrier relaxation in nanoparticles. If we ignore non-linear dynamic processes for now (to be discussed in the next section), the mechanisms are relatively simple and straightforward. Electronic 
relaxation in nanoparticles is similar to that observed in bulk solids, except with the important complication involving trap states.

For simplicity, let us first ignore trap states and suppose that there is at most one exciton or electron-hole pair per nanoparticle. In this simple case, above bandgap excitation produces an exciton or an electron in the $\mathrm{CB}$ and hole in the VB bound to each other by Columbic attraction. If the electron and/or hole have excess kinetic energy, they will first relax to the bandedge (the electron to the bottom of the CB and the hole to the top of the VB) through electron-phonon interactions on the tens to hundreds of fs time scale. Subsequently, the relaxed electron and hole at the bandedge can recombine radiatively, producing $\mathrm{PL}$, or non-radiatively, usually producing heat. In a perfect crystal with few or no defects and hence a very low trap state density, radiative recombination dominates. The PL quantum yield in this case is very high, near $90 \%$ or more.

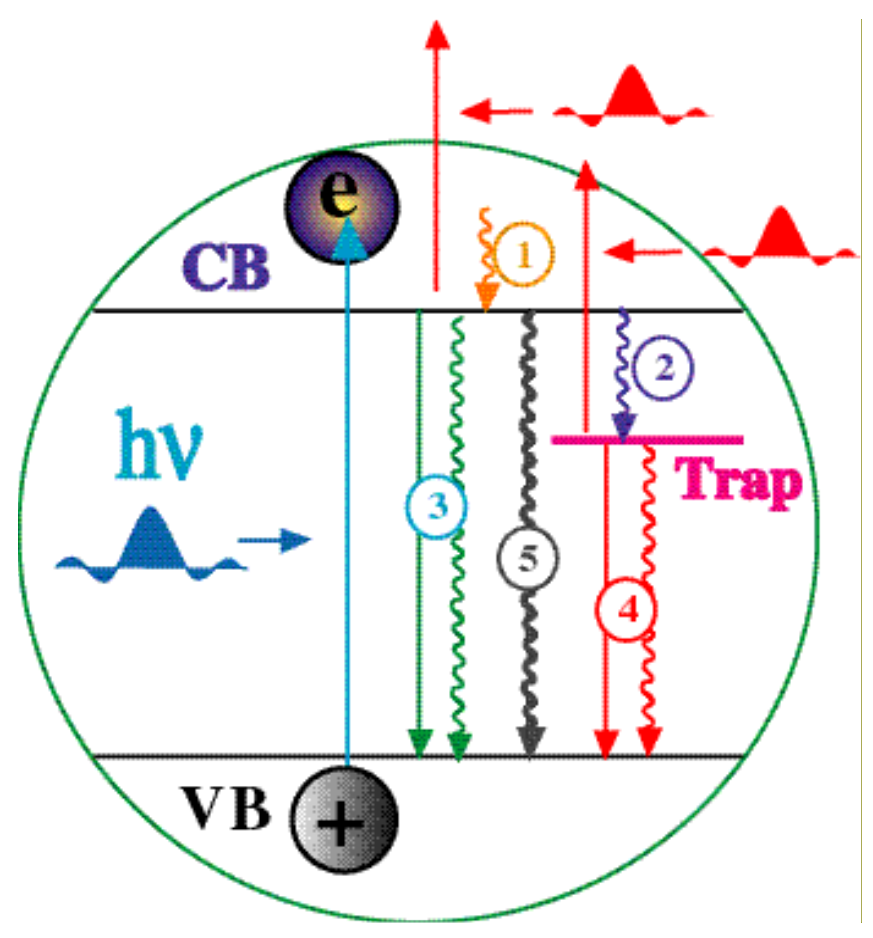

Figure 3. Illustration of a pump-probe approach for lifetime measurements. Different electronic relaxation pathways in a nanoparticle with trap states are illustrated: 1) electron relaxation through electron-phonon coupling with the conduction band (likewise for the hole in the valence band) following excitation across the bandgap; 2) trapping of electrons into trap states due to defects or surface states; 3) radiative and non-radiative bandedge electron-hole (or exciton) recombination; 4) radiative and non-radiative trapped electron-hole (or slightly relaxed exciton) recombination; and 5) non-linear and non-radiative excitonexciton annihilation. 
However, in a crystal with defects or a QD with a relatively high density of bandgap trap states due to surface or internal defects, trapping of electrons or holes into these states becomes significant. In many cases, trapping of charge carriers is faster than bandedge radiative recombination and therefore trapping dominates. Typically, the higher the density of trap states, the more likely and faster trapping will take place. Trapping lowers the bandedge PL yield and often the overall PL yield of the sample.

Following trapping, charge carriers (electrons, holes, or both) can either undergo further trapping (e.g. from shallow to deeper traps, not illustrated in Figure 3) or recombine radiatively or non-radiatively, as illustrated in Figure 3. This recombination process is quite similar in nature to bandedge carrier recombination processes. If this step is radiative, PL from trap states can be observed, red-shifted relative to bandedge PL since the trapping process causes non-radiative energy loss. Therefore, bandedge or trap state PL can be differentiated. If time-resolved PL is used, one can determine the lifetime of the bandedge states versus trap states. Since PL is a very sensitive technique, it is very useful for probing and understanding optical, dynamic, and electronic structure of nanomaterials.

With low excitation intensity, most nanoparticles will only have one exciton per particle, and no non-linear processes, such as exciton-exciton annihilation or Auger recombination, should occur. In this case, the charge carrier relaxation dynamics are relatively simple. Following relaxation within the conduction band for the electron and valence band for the hole, the carriers at or near the bandedge will recombine either radiatively or non-radiatively. The observed lifetime, $\tau_{\mathrm{ob}}$, is related to the radiative, $\tau_{\mathrm{r}}$, and non-radiative, $\tau_{\mathrm{nr}}$, lifetimes, by the following equation:

$$
1 / \tau_{\mathrm{ob}}=1 / \tau_{\mathrm{r}}+1 / \tau_{\mathrm{nr}}
$$

The lifetimes are related to the PL quantum yield, $\Phi_{\mathrm{PL}}$, by:

$$
\Phi_{\mathrm{PL}}=\tau_{\mathrm{ob}} / \tau_{\mathrm{r}} \text {. }
$$

There is sometimes confusion between radiative lifetime $\left(\tau_{r}\right)$ and observed lifetime $\left(\tau_{\mathrm{ob}}\right)$. These two are strictly speaking equal only in the limit that $\tau_{\mathrm{nr}}$ is very long or the PL quantum yield is nearly $100 \%$, as in a perfect single crystal, according to equations 3 and 4 . Any lifetime measured experimentally based on time resolved PL or TA measurements is just the observed lifetime $\tau_{\mathrm{ob}}$, which contains contributions from both $\tau_{\mathrm{r}}$ and $\tau_{\mathrm{nr}}$.

In nanoparticles, oftentimes there is a high density of trap states that lead to charge carrier trapping before or following relaxation in the $\mathrm{CB}$ or VB. The trapped carriers can recombine radiatively or non-radiatively, similar to bandedge carrier recombination. Trapping is typically a nonradiative process and contributes to $\tau_{\mathrm{nr}}$ in equation 3 . Also, there is usually a distribution of trap states and trapping can therefore be through several stages, e.g. through first shallow traps and then deep traps, thus complicating the kinetics. The shallow traps usually have shorter observed lifetimes than deep traps. The nature of trap states depends on the chemical nature, crystal structure, and details of surface characteristics. 
Surface-related trap states can be manipulated and this is often reflected sensitively in PL changes (spectral position and intensity). For example, based on time-resolved studies of a number of systems, the following general observations have been made: i) a high density of trap states corresponds to overall low PL yield; ii) a high density of trap states corresponds to relatively strong PL from trap states and weak or no PL from bandedge states; iii) a high density of trap states corresponds to short observed lifetime of charge carriers or the exciton; iv) a high density of trap states corresponds to a higher threshold for non-linear processes since it is harder to saturate all of the trap states.

The last statement implies that nanomaterials are better non-linear optical materials in the sense that they can tolerate a higher density of optical and possibly other radiation. This could be very useful for radiation protection applications.

In the scenario where the nanoparticles have no trap states within the bandgap at all, it is essentially a small perfect single crystal. In this ideal case, which is challenging to achieve experimentally, the behavior of the exciton or charge carrier is similar to that in bulk single crystals with the difference of spatial confinement. This would in principle allow for study of the pure spatial confinement effect without any influence from trap or surface states. However, this is not easy to achieve in reality due to bandgap states that are challenging to remove completely.

\subsection{Non-linear Dynamic Properties}

Non-linear behavior occurs when there are multiple excitons generated in the same spatial region at the same time where there is strong interaction between the excitons. This is typically reflected as a dynamic process that depends non-linearly (e.g. quadratically or even higher order) on the excitation light intensity [6,7,71,86, 180-182].

There have been various explanations for the observation of non-linear dynamical behavior in nanomaterials, including higher order kinetics, Auger recombination, and exciton-exciton annihilation (illustrated in Figure 4). It is challenging to assign an exact mechanism from only experimental data. All these models can explain the observations reasonably well. We have favored the exciton-exciton annihilation model since Auger recombination involves ionization and most time-resolved studies do not provide direct evidence for charge ejection. In the excitonexciton annihilation model, high excitation laser intensity for the pump pulse produces multiple excitons per particle that can interact and annihilate, resulting in one exciton doubly excited and another one deexcited. If the rate of trapping is faster than the rate of exciton-exciton annihilation, which is often the case, trapping will reduce the probability of exciton-exciton annihilation. However, when trap states are saturated, exciton-exciton annihilation will take place. Therefore, nanoparticles with a higher density of trap states have a higher threshold for observing 
exciton-exciton annihilation or require higher pump laser intensities to observe this non-linear process. This behavior has been clearly demonstrated in $\mathrm{CdS}$ nanoparticles $[60,86]$.

The comparisons can be subtle and require careful attention when different sized or shaped nanoparticles are considered. This is partly because there are several factors, some competing, that need to be accounted for while making a comparison [183]. For example, when particles of different sizes but the same number of excitons per particle are compared, the smaller particles show a stronger non-linear effect or, conversely, a lower excitation threshold for observing the non-linear process. This is because smaller particles have stronger spatial confinement and lower density of states per particle that both facilitate exciton-exciton annihilation. On the other hand, when two samples of the same material such as $\mathrm{CdS}$ with the same nominal optical density or concentration but different particle sizes are compared under the same excitation intensity, the larger particles show stronger non-liner effect. This is apparently due to a larger number of excitons per particle for the larger particles. This indicates that the volume factor dominates over the effect of trap states. In other words, larger particles have a larger molar absorptivity (see the section on linear optical properties) and thus absorb more photons to create more excitons for a given laser pulse. The observation is opposite to what is expected for a larger number of trap states per particle, which for the larger particle should raise the threshold and thereby suppress exciton-exciton annihilation. These are illustrated schematically in Figure 5

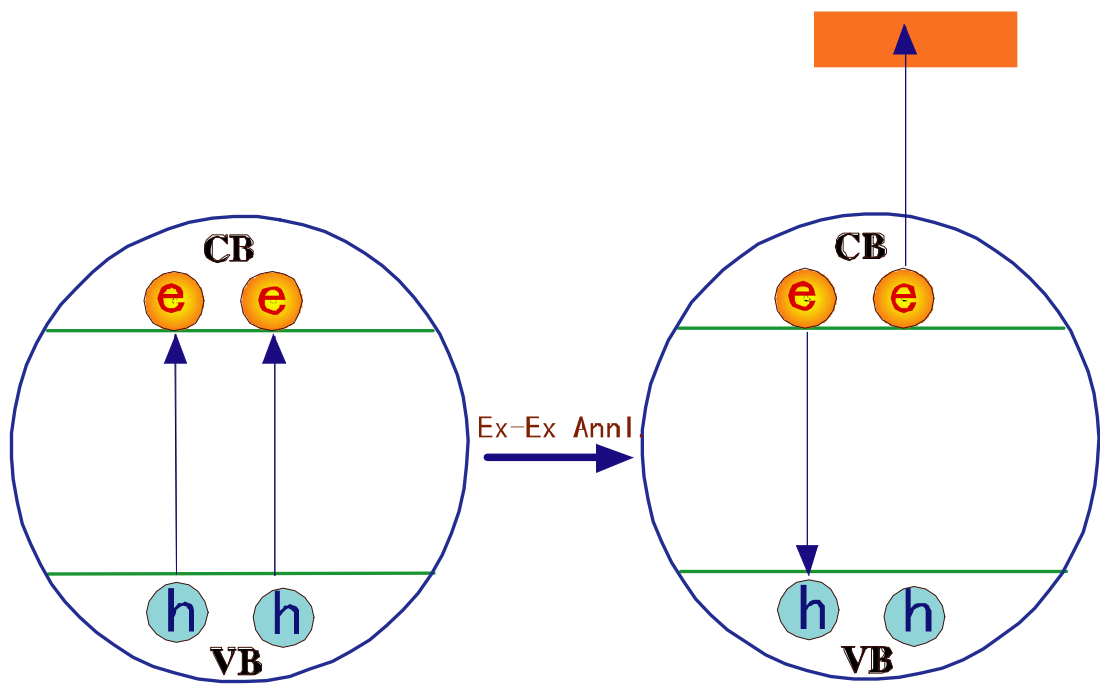

Figure 4. Illustration of non-linear and non-radiative exciton-exciton annihilation that results in the non-radiative de-excitation or recombination of one exciton and excitation of the other exciton to higher energy. The excited exciton will eventually relax radiatively or non-radiatively. 
To further support the above argument, we have found that particles with similar volume but different shapes and thereby a different density/distribution of trap states show different thresholds for non-linear effects. For non-spherical particles, the PL yield is much lower compared to that of spherical particles, indicating a higher density of trap states for non-spherical particles. Based on the model discussed above, we should expect non-spherical particles to show a higher threshold for or weaker effect of exciton-exciton annihilation since it is harder to achieve trap state saturation. This is completely consistent with the experimental observation of stronger non-linear effect for the spherical particles [183].

It should be pointed out that our observations are qualitatively consistent with that made by Klimov et al. on CdSe nanoparticles [182]. However, the time constant for non-linear decay is much faster in our observation (a few ps) than that reported by Klimov et al. (as long as hundreds of ps). It is unclear if this difference is due to differences in the systems studied, experimental conditions, or even simply due to differences in data analysis and interpretation. Further studies are needed to better understand this issue.

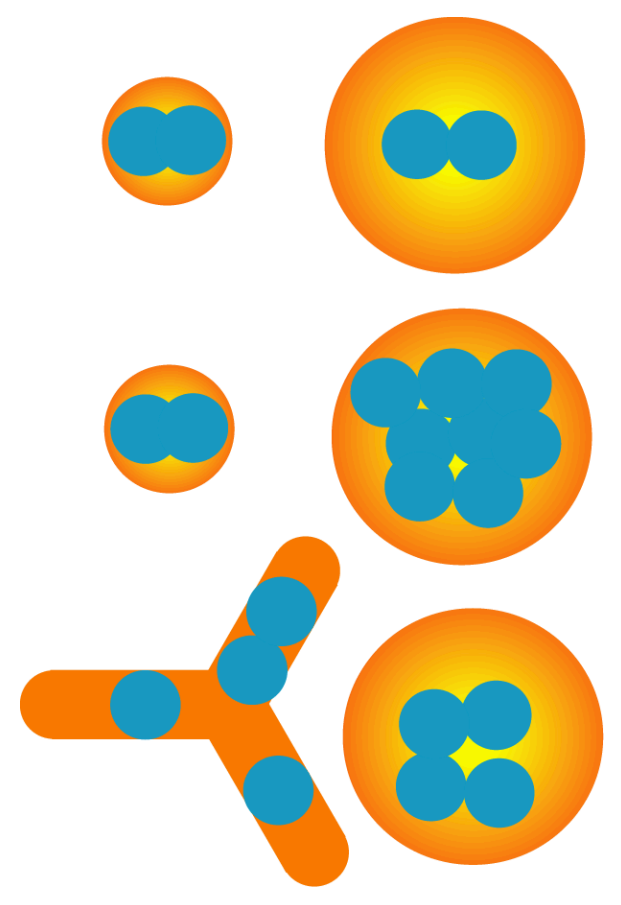

Figure 5. Illustration of the size and shape dependence of exciton-exciton annihilation. 
Very recently, it has been suggested that multiple excitons can be generated using a single photon in small bandgap semiconductors such as $\mathrm{PbSe}$ and $\mathrm{PbS}$ [184-189]. This is potentially useful for solar energy conversion and other applications. Even though this is theoretically possible and there is preliminary supporting experimental evidence, it is unclear what the probability or efficiency is in practice. Given that electronic relaxation is typically very fast (less than $100 \mathrm{fs}$ ), this multiexciton generation process with one photon is likely to be inefficient or has a very small cross section unless the energy levels involved can be carefully and intelligently designed to enhance the process. Impact ionization is a scheme proposed for enhancing multiple exciton generation (MEG) [184-188]. It is yet to be realized in practical device applications. Further research is needed to verify the feasibility of this approach and strategies to realize or enhance it.

\subsection{Charge Transfer Dynamics Involving Nanoparticles}

Beside dynamic processes of charge carrier relaxation in nanoparticles, other important dynamic processes of interest include charge transfer from nanoparticles to other species such as surface attached molecules or from molecules to nanoparticles (often referred to as charge injection). For example, dye molecules have been used to sensitize metal oxide nanoparticles such as $\mathrm{TiO}_{2}$ and $\mathrm{ZnO}$ for potential solar cell applications [190-195]. In this case, one important process is electron injection from the dye molecule to the nanoparticle. The rate of injection is one critical factor in determining the solar conversion efficiency. The rate itself depends on a number of factors including the relative electronic energy levels of the dye and nanoparticles, strength of interaction between them, optical properties of the dye. Time-resolved studies have been successfully used to determine the rate of charge injection and its dependence on various factors such as distance and coupling strength [196]. In general, the injection rate has been found to be very fast, on the time scale of $100 \mathrm{fs}$ or less [191].

Charge transfer from nanoparticles to molecules near or on the nanoparticles has also been studied using time-resolved techniques. Study of such processes is important for understanding photochemical and photocatalytic reactions when nanoparticles serve as photocatalysts or catalysts. For instance, electron transfer dynamics from $\mathrm{CdS}$ and $\mathrm{CdSe}$ NPs to electron acceptors, e.g. viologen derivatives, adsorbed on the particle surface have been studied using transient absorption, transient bleach and time-resolved fluorescence [197, 198]. Electron transfer was found to take place on the time scale of 200-300 fs and competes effectively with trapping and electron-hole recombination. These results are important to understanding interfacial charge transfer involved in photocatalysis and photoelectrochemistry applications. 


\section{Doped Semiconductor Nanomaterials}

Doping is a powerful and effective way to alter the electronic and optical properties of a semiconductor. Doping is essential in the semiconductor industry since most semiconductors including silicon are essentially insulators without doping at room temperature.

Similar to bulk materials, doping has been used for semiconductor nanomaterials $[45,46]$. There are some unique challenges with doping nanomaterials. For example, when the size is very small, one dopant ion per nanostructure can make a major difference in the properties of the nanostructure The addition of the dopant can introduce electronic and/or structural defects into the pristine nanomaterial that can be advantageous or deleterious. It is therefore critical to attempt to dope the nanostructures uniformly, i.e. same number of dopant ions per nanostructure (e.g. Figure 6). There are further complications to this issue beyond just the number of dopants. For example, the location of the dopant on the surface versus the interior affects the optical or electrical properties differently. Another issue is the interaction among the dopants when the dopant concentration per particle is high, e.g. two or more dopants in close proximity. This will remain a challenging and interesting issue for years to come, particularly when spatial features become smaller and the importance of the dopant becomes more critical.

Recently, there have been some reports of uniform doping using either growth or nucleation doping techniques by decoupling the doping and growth processes. Briefly, in nucleation doping reaction conditions are controlled in such a way along with judicious choice of reactants that a nucleus of dopants such as MnSe can be created followed by shell growth of $\mathrm{ZnSe}$ effectively confining the dopants to the center of the particle. Alternatively, also in a similar manner growth doping starts by creating a small $\mathrm{ZnSe}$ host crystal and then the doping atom may be introduced to either controllably dope the surface or the interior [199, 200].

Almost all studies of doped semiconductor nanostructures have been performed on ensemble averaged samples, i.e. the sample contains particles with a distribution of dopant per particle. Doping typically follows a Poisson distribution. The measured results need to be interpreted in such a manner. Several good review articles on doped semiconductor nanomaterials have appeared recently [5, 45, 46]. In this article, we will show a few examples to highlight the complexity and uniqueness of doped semiconductor nanoparticles. 

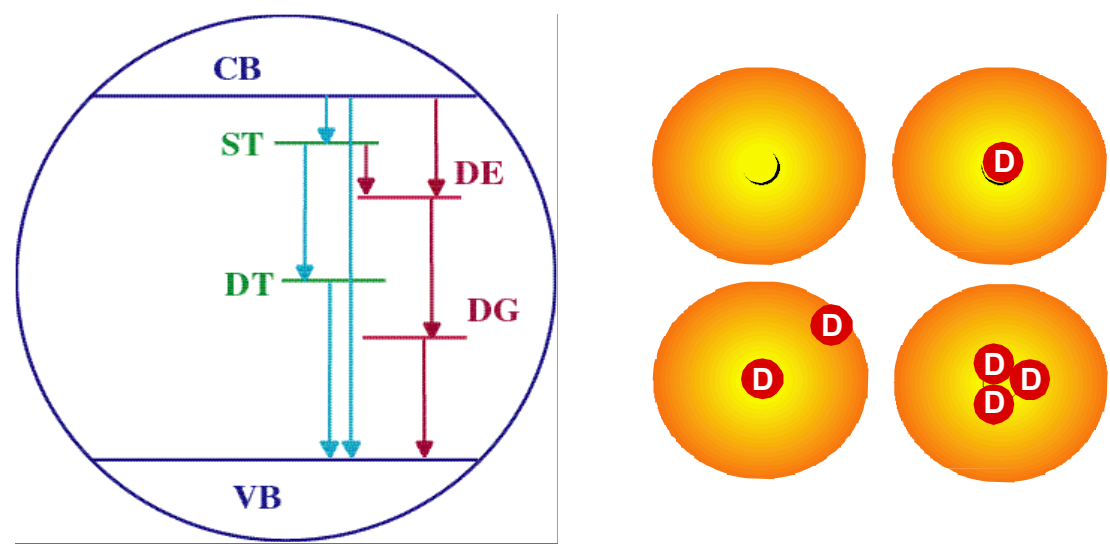

Figure 6. Left: Schematic illustration of energy levels of shallow trap (ST), deep trap (DT), dopant excited state (DE) and dopant ground state (DG) in a doped semiconductor nanoparticle with respect to the band edges of the valence band (VB) and conduction band (CB). Right: Illustration of nanoparticles with different numbers of dopant ions per particles as well as different locations of the dopant ions in the nanoparticles.

One of the most extensively studied doped nanoparticles is Mn doped $\mathrm{ZnS}$, which is of interest for applications as a phosphor material [201-207]. Related systems studied recently include ZnSe:Mn that clearly show a strong correlation between optical emission and the location of the Mn dopant ion [208]. It has been found that at 1\% Mn doping (measured in term of starting reactant materials, but actually determined for the product particles), the $\mathrm{ZnSe}$ bandedge PL is significantly quenched, by over a factor of 100 in peak intensity in comparison (Figure 7). It is clear that with roughly one $\mathrm{Mn}^{2+}$ ion per nanoparticle, significant PL quenching of the host $\mathrm{ZnSe}$ occurs. This makes sense since the time-integrated PL measured is conducted at low excitation intensity and is a single electron transition event. One $\mathrm{Mn}$ ion per particle can affect the relaxation pathways dramatically. Surprisingly, however, no PL from the Mn dopant ion was observed at this $1 \%$ doping level as one would expect. It was found that at this particular doping level, the Mn ions are primarily on the surface of the $\mathrm{ZnSe}$ nanoparticles and are consequently non-emissive.

This conclusion was reached after using a combination of PL, ESR, and XAFS on many different samples with varying doping levels. XAFS was able to provide a direct measure of the location and coordination environment of the different ions including $\mathrm{Zn}, \mathrm{Se}$, and Mn [208]. At 6\% Mn doping, the ZnSe bandedge PL was further quenched by a factor of 800 relative to the undoped material with the characteristic $580 \mathrm{~nm}$ Mn dopant emission observed as expected. At this doping level, ESR clearly shows two different environments for Mn ions with XAFS data indicating two different coordination sites and symmetry, octahedron versus tetrahedron 
[208]. It was suggested that the tetrahedron $\mathrm{Mn}$ site is in the interior of the $\mathrm{ZnSe}$ nanoparticle with $\mathrm{Mn}$ substituting for the $\mathrm{Zn}$ cations while the octahedral $\mathrm{Mn}$ site is located at or near the $\mathrm{ZnSe}$ nanoparticle surface. While the interior $\mathrm{Mn}$ is emissive, the surface site is non-emissive, as illustrated in Figure 8, possibly because the extra ligands on the surface can potentially interact with either the capping agent or the solvent quenching the Mn emission. If this model is correct, it suggests that one should avoid having surface $\mathrm{Mn}$ in applications such as nanophosphors where $\mathrm{Mn}$ emission is desired. One needs to either remove surface Mn ions or find ways to encapsulate the $\mathrm{Mn}$ ions perhaps via a shell of a wide bandgap semiconductor such as $\mathrm{ZnS}$ into the lattice so they become optically emissive. Growth or nucleation growth, as discussed earlier, may be a way help solve this problem of encapsulation of dopants [199, 200].

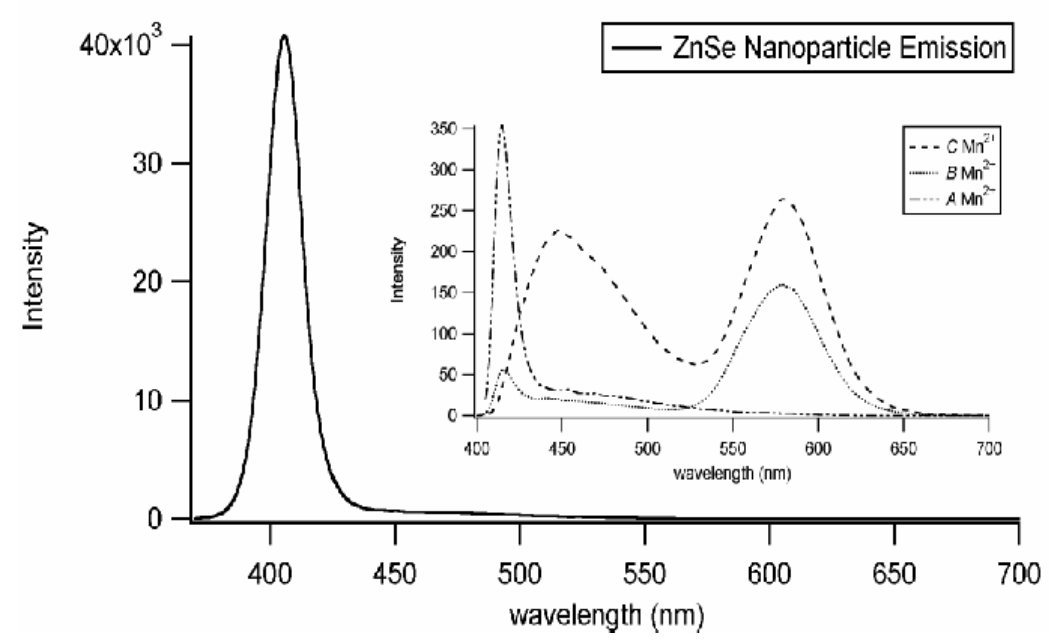

Figure 7. Photoluminescence spectra of $\mathrm{ZnSe}: \mathrm{Mn}$ with different Mn doping levels: $1,1,5$ and $10 \%$ (Reproduced with permission from ref. [208]).

Even though metal oxides are often considered insulators, in the context of this discussion, doped metal oxides share many similar properties to semiconductors as they can be considered large bandgap semiconductors. One very interesting doped metal oxide is $\mathrm{ZnO}$ nanoparticles doped with various ions such as $\mathrm{Co}^{2+}[209], \mathrm{Mn}^{2+}[210,211]$, and $\mathrm{Cu}^{2+}$ [212]. As high as $35 \%$ of Co can be doped into $\mathrm{Co}_{\mathrm{x}} \mathrm{Zn}_{1-\mathrm{x}} \mathrm{O}$ thin films without phase segregation [209]. As another example, $\mathrm{TiO}_{2}$ nanoparticles and nanotubes have been doped with non-metal ions such as $\mathrm{N}$ to extend their photoresponse to the visible region and improve photoactivity [213-216]. Both $\mathrm{TiO}_{2}: \mathrm{N}$ and $\mathrm{ZnO}: \mathrm{N}$ have found success in narrowing the bandgap and increasing light harvesting efficiency [217, 218]. In addition, research has been conducted on the photoelectrochemical properties and photocatalytic activity for solar energy applications. Several different synthesis protocols have been developed to produce $\mathrm{TiO}_{2}: \mathrm{N}$. The usual doping process involves using 
ammonia as a nitrogen source by sol-gel, thermal, or hydrothermal chemical methods [219, 220].

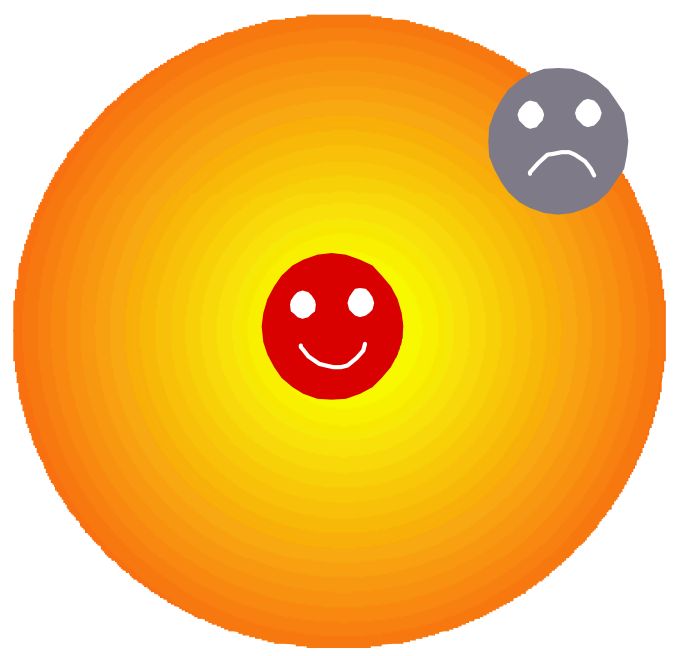

Figure 8. Schematic illustration of two different $\mathrm{Mn}^{2+}$ sites in $\mathrm{ZnSe}$ nanoparticles with different coordination symmetry and optical properties: tetrahedral and emission at $580 \mathrm{~nm}$ for the interior site while octahedral and non-emissive for the surface site.

Charge carrier dynamics in doped semiconductor nanomaterials have also been studied with emphasis often placed on the carrier lifetime in the excited electronic state of the dopant ions. Such lifetimes can vary significantly from dopant to dopant, ranging from ns to $\mathrm{ms}$. Some of the interesting and unresolved issues include how trap states mediate the rate of energy or charge transfer from host to dopant. It can be expected that the trap state could play an important role in energy or charge transfer processes especially when the trap states are located in between the donor states of the host and the acceptor states of the dopant. These issues require further investigation.

In some cases, the study can be complicated by host trap states, especially when there is spectral overlap between trap state and dopant transitions. For example, in the well-studied case of $\mathrm{ZnS}: \mathrm{Mn}$, there had been some controversy as well as some confusion about the carrier lifetime and related PL yield. In 1994, it was first reported that the PL lifetime of $\mathrm{Mn}^{2+}$ in $\mathrm{ZnS}: \mathrm{Mn}$ nanoparticles was significantly shorter ( 20 ns) than that in the bulk and had a greater luminescence efficiency [221-223]. The observed ns decays were five orders of magnitude shorter than the bulk luminescence lifetime $(1.8 \mathrm{~ms})$ [224]. This was explained using rehybridization between the s-p conduction band of $\mathrm{ZnS}$ and the $3 \mathrm{~d}$ states of the $\mathrm{Mn}^{2+}$ because of quantum confinement. However, subsequent studies have shown that the $\mathrm{Mn}^{2+} \mathrm{PL}$ lifetime in $\mathrm{ZnS}$ nanoparticles is the same as the bulk (1.8 ms) [225-228]. In our study of the PL kinetics of 
$\mathrm{ZnS}: \mathrm{Mn}$ nanoparticles monitored at $580 \mathrm{~nm}$, we observed a slow $1.8 \mathrm{~ms}$ decay that is similar to the $\mathrm{Mn}^{2+}$ emission lifetime in bulk $\mathrm{ZnS}$ as well as fast ns and $\mu$ s decays that are also present in undoped $\mathrm{ZnS}$ particles and thereby attributed to trap state emission [227], as shown in Figure 9.

Although these recent studies have consistently shown that the $\mathrm{Mn}^{2+}$ PL lifetime is similar in nanoparticles as in bulk $\mathrm{ZnS}: \mathrm{Mn}$, there are still active discussion over whether the PL quantum yield is higher in nanoparticles than in bulk. Recent studies [201-206], including a theoretical study [207], made claims of enhancement that seem to support the original claim of enhanced PL [222]. For instance, a study on the PL and EL properties of $\mathrm{ZnS}: \mathrm{Mn}$ nanoparticles has found that the PL efficiency increased with decreasing particle size [229]. However, most of the reported PL or EL quantum yields have not been quantitatively compared between nanoparticles and bulk. Quantitative and calibrated measurements of the PL quantum yield are essential to establish if there is truly an enhancement in nanoparticles.
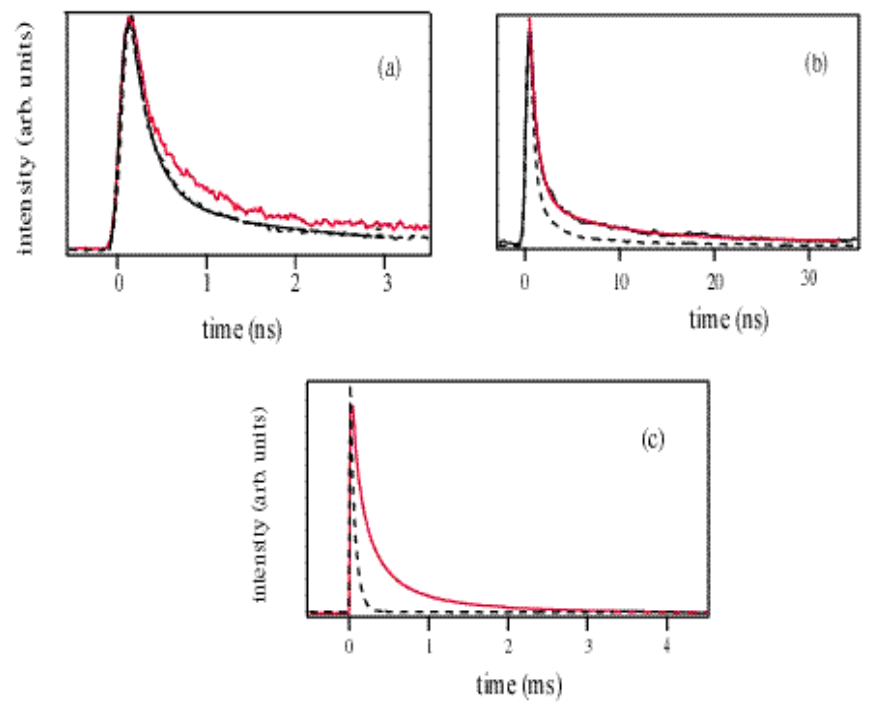

Figure 9 PL delay kinetics monitored at $580 \mathrm{~nm}$ of Mn-doped (solid red) and undoped (dashed black) $\mathrm{ZnS}$ nanoparticles on different time scales. Adapted with permission from ref. [227]. The major difference on long time scales is due to $\mathrm{Mn}$ doping.

Another time resolved study that has come out of our lab is a comparative study between same diameter ZnSe:Mn with ZnSe using picosecond PL in combination with femtosecond transient absorption [230]. Briefly, it was shown that the electronic relaxation as determined from fs spectroscopy was multi-exponential and overall faster in Mn doped $\mathrm{ZnSe}$ particles relative to undoped $\mathrm{ZnSe}$. Also of interest is that PL relaxation was also multi-exponential in nature and that in the doped material the overall PL decayed more rapidly then the undoped $\mathrm{ZnSe}$. In addition on all 
time scales the relaxation was faster in $\mathrm{ZnSe:Mn}$ than in $\mathrm{ZnSe}$. The difference in lifetimes on all time scales was attributed to a mechanism of energy transfer from host to dopant either mediated by trap states and another without (directly from the bandedge states). From these results the energy transfer possibly occurs on a time scale of tens to hundreds of ps which appears to be shorter than that reported in a study of $\mathrm{ZnS}: \mathrm{Mn}$ by Chung where they found a rise time for the $\mathrm{Mn}^{2+}$ luminescence of $700 \mathrm{ps}$ [228].

\section{Applications of Optical Properties}

While this chapter focuses on fundamental optical and dynamic properties of nanomaterials, we wish to briefly discuss some of the relevant emerging technological applications that span a wide range of fields from chemical sensing, photocatalysis, photoelectrochemistry, solar energy conversion, to biomedical detection and therapy. Most of these applications take advantage of some or all of the following unique features of nanomaterials: i) nanoscale sizes are comparable to carrier scattering lengths, this significantly reduces the scattering rate, thus increasing the carrier collection efficiency; ii) nanoparticles have strong optical absorption coefficient due to increased oscillator strength; iii) by varying the size, nanoparticle bandgap can be tuned to absorb in a particular wavelength region, possibly covering the entire solar spectrum; iv) nanoparticle-based devices can be built on flexible substrates; v) nanoparticle-based devices can be lightweight and easy to make at potentially low cost; vi) nanomaterials have large surface-to-volume ratio and the surface can be modified and tailored for specific applications; vii) nanomaterials lend themselves conveniently for integration and assembly into larger and more sophisticated systems.

Of course, nanomaterials also have limitations when it comes to specific applications. For example, the large surface area of nanomaterials makes them vulnerable for surface defects and trap states that could have adverse effect on optical and other properties. Large particle surface areas potentially make nanoparticles more reactive due to dangling bonds. Even in carefully prepared, high quality samples, the density of trap states tend to be much higher than corresponding bulk materials and this results in low mobility or conductivity of charge carriers, which is undesirable for applications requiring good charge carrier transport. Some of these limitations can be overcome by careful design and engineering of the overall devices structures.

A few application examples will be given below in connection to the optical properties of nanomaterials. 


\subsection{Energy Conversion: Photovoltaics and Photoelectrochemistry}

Solar energy conversion into electricity or chemical energy such as hydrogen represents one of the most promising applications of optical properties of nanomaterials $[190,192-195,231]$. For example, dyesensitized solar cells have attracted significant attention since the initial report in 1991 of a power conversion efficiency of $12 \%$ [190]. Other variations of solar cells based on nanomaterials have also been demonstrated. While there are still issues related to efficiency, lifetime, and cost, they look very encouraging. While $0-\mathrm{D}$ nanomaterials offer the largest surface-to-volume ratio that is often desired for solar cell applications, charge carrier transport is usually poor or mobility is low due to trapping of carrier by surface or other defect states and the need of carrier hopping for conduction. In this regard, 1-D or 2-D nanomaterials should offer better transport properties over 0-D nanomaterials. Of course, the surface-to-volume ratio is somewhat compromised.

There is growing recent interest in using various 1-D structures for solar cell applications, including $\mathrm{CdSe}$, dye-sensitized $\mathrm{TiO}_{2}$ and $\mathrm{ZnO}$ nanorods, nanowires, and nanotubes [232-239]. In many cases, improved photovoltaic performance has been found compared to 0-D nanoparticle films. However, most of these nanostructures are not as well aligned or ordered as one would like. The order or alignment of the 1-D structures should further improve their performance. Fabrication of well-ordered 1-D nanostructure arrays usually requires more sophisticated fabrication or synthesis techniques such as glancing angle deposition (GLAD) [240, 241]. Figure 10 shows an example of an idealized solar cell structure based on 1D nanostructures.

Related to solar energy conversion directly into electricity is photocatalysis and photoelectrochemistry (PEC) that converts solar energy into chemical fuels such as hydrogen. One example is PEC conversion of water into hydrogen which is of strong current interest. In PEC, light illuminates one electrode (photoanode) that generates an electron and hole pair. The hole reacts with water molecules to produce $\mathrm{O}_{2}$ while the electron is transported to another electrode (cathode, usually a metal such as $\mathrm{Pt}$ ) where it reacts with protons $\left(\mathrm{H}^{+}\right)$to produce $\mathrm{H}_{2}$ gas. For most materials, the process requires an external bias in the 0 to $1 \mathrm{~V}$ range to assist in the conversion process and can be supplied by a battery or even a photovoltaic cell. The overall $\mathrm{H}_{2}$ generation efficiency depends on a number of factors, most importantly the structural, chemical, and energetic characteristics of the cathode material that are often metal oxides or other semiconductors. The electrode materials can be bulk materials, thin films, or nanomaterials. Similar to solar cells, the issues of carrier transport versus surface area are often factors to consider when assessing their performances. 1-D materials again might hold some promise due to the combination of good electrical transport properties and large surface areas. 
It is important to develop inexpensive techniques for producing ordered or aligned 1-D structures.

\subsection{Photochemistry and Photocatalysis}

Nanomaterials have played a critical role in many important chemical reactions as reactants, catalysts, or photocatalysts. In relation to the optical properties that are of interest to this chapter, we will discuss briefly photochemical and photocatalytic reactions involving nanomaterials as reactants or photocatalysts. Their reactivities are often altered or enhanced due to size dependent changes in their redox potentials and high density of active surface states associated with a very large surface-to-volume ratio.

It has also been demonstrated that photooxidation of some small molecules on semiconductor nanoparticles can lead to the formation of biologically important molecules such as amino acids, peptide oligomers, and nucleic acids [242, 243]. In addition to photooxidation, photoreduction based on semiconductor nanoparticles have also been explored for synthesis of organic molecules [244, 245]. For example, photoinduced reduction of $p$-dinitrobezene and its derivatives on $\mathrm{TiO}_{2}$ particles in the presence of a primary alcohol has been found to lead to the formation of benzimidoles with high yields [246].

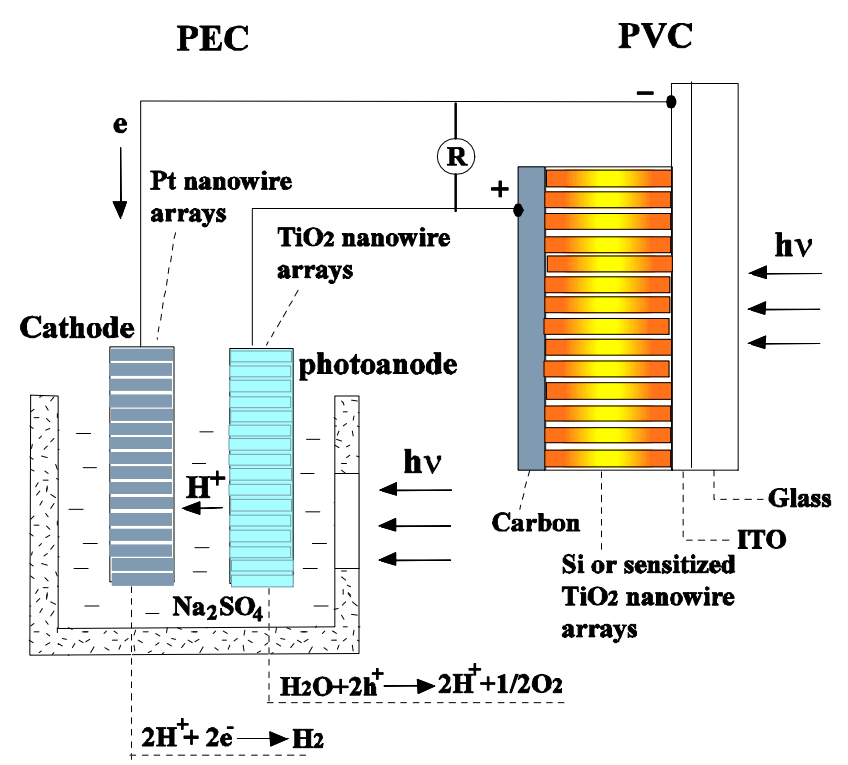

Figure 10. Schematic of an integrated photovoltaic cell (PVC) with a photoelectrochemical cell (PEC) for hydrogen generation from water splitting using 1-D nanostructures for both the PVC and PEC.

Another important area of application for semiconductor nanoparticles is in photoelectrochemical reactions. Similar to photochemical reactivities, the photoelectrochemical properties of nanoparticles are often quite 
different from those of their bulk counterparts. For example, structurally controlled generation of photocurrents has been demonstrated for doublestranded DNA-cross-linked CdS nanoparticle arrays upon irradiation with light [247]. The electrostatic binding of $\left[\mathrm{Ru}\left(\mathrm{NH}_{3}\right)_{6}\right]^{3+}$ to ds-DNA units provides tunneling routes for the conduction band electrons and thus results in enhanced photocurrents. This could be useful for DNA sensing applications. Photoelectrochemical behavior has been demonstrated in a number of semiconductor nanoparticle films, including $\mathrm{CdS}$ and $\mathrm{CdSe}$ [248-250], $\mathrm{ZnO}$ [251], $\mathrm{TiO}_{2}$ [252-255], Mn-doped $\mathrm{ZnS}$ [256, 257], $\mathrm{WO}_{3}$ [258], $\mathrm{SnO}_{2} / \mathrm{TiO}_{2}$ composite [259], and $\mathrm{TiO}_{2} / \mathrm{In}_{2} \mathrm{O}_{3}$ composite [260]. The large bandgap semiconductors, e.g. $\mathrm{TiO}_{2}$ and $\mathrm{ZnO}$, often require sensitization with dye molecules so that photoresponse can be extended to the visible region of the spectrum [191, 252, 256].

Photocatalysis based on semiconductors plays an important role in chemical reactions of small inorganic, large organic, and biological molecules. The photocatalytic reactivities are strongly dependent on the nature and properties of the photocatalysts, including $\mathrm{pH}$ of the solution, particle size, and surface characteristics [261]. These properties are sensitive to preparation methods [261]. Impurities or dopants can significantly affect these properties as well as reactivities. For example, it has been shown that selectively doped nanoparticles have a much greater photoreactivity as measured by their quantum efficiency for oxidation and reduction than their undoped counterparts [262]. A systematic study of the effects of over 20 different metal ion dopants on the photochemical reactivity of $\mathrm{TiO}_{2}$ colloids with respect to both chloroform oxidation and carbon tetrachloride reduction has been conducted [262, 263]. A maximum enhancement of 18 -fold for $\mathrm{CCl}_{4}$ reduction and 15-fold for $\mathrm{CHCl}_{3}$ oxidation in quantum efficiency for $\mathrm{Fe}(\mathrm{III})$-doped $\mathrm{TiO}_{2}$ colloids have been observed [264]. Recent studies have shown that the surface photovoltage spectra (SPS) of $\mathrm{TiO}_{2}$ and $\mathrm{ZnO}$ nanoparticles can be an effective method for evaluating the photocatalytic activity of semiconductor materials since it can provide a rapid, non-destructive monitor of the semiconductor surface properties such as surface band bending, surface and bulk carrier recombination and surface states [265]. It has been demonstrated that the weaker the surface photovoltage signal is, the higher the photocatalytic activity is in the case of nanosized semiconductor photocatalysts.

Photocatalytic oxidation of organic and biological molecules is of great interest for environmental applications, especially in the destruction of hazardous wastes. The ideal outcome is complete mineralization of the organic or biological compounds, including aliphatic and aromatic chlorinated hydrocarbons, into small inorganic, non- or less- hazardous molecules, such as $\mathrm{CO}_{2}, \mathrm{H}_{2} \mathrm{O}, \mathrm{HCl}, \mathrm{HBr}, \mathrm{SO}_{4}{ }^{2-}$, and $\mathrm{NO}_{3}{ }^{-}$. Photocatalysts include various metal oxide semiconductors, such as $\mathrm{TiO}_{2}$, in both bulk and particulate forms. Compounds that have been degraded by semiconductor photocatalysis include alkanes, haloalkanes, aliphatic alcohols, carboxylic 
acids, alkenes, aromatics, haloaromatics, polymers, surfactants, herbicides, pesticides and dyes, as summarized in an excellent review article by Hoffmann [264]. It has been found in many cases that the colloidal particles show new or improved photocatalytic reactivities over their bulk counterparts.

One of the most important areas of application of photocatalytic reactions is removal or destruction of contaminates in water treatment or purification [266-268]. Major pollutants in waste waters are organic compounds. Small quantities of toxic and precious metal ions or complexes are usually also present. As discussed above, semiconductor nanoparticles, most often $\mathrm{TiO}_{2}$, offer an attractive system for degrading both organic and inorganic pollutants in water. Water treatment based on photocatalysis provides an important alternative to other advanced oxidation technologies such as $\mathrm{UV}-\mathrm{H}_{2} \mathrm{O}_{2}$ and $\mathrm{UV}-\mathrm{O}_{3}$ designed for environmental remediation by oxidative mineralization. The photocatalytic mineralization of organic compounds in aqueous media typically proceeds through the formation of a series of intermediates of progressively higher oxygen to carbon ratios. For example, photodegradation of phenols yields hydroquinone, catechol and benzoquinone as the major intermediates that are eventually oxidized quantitatively to carbon dioxide and water [269].

In general, the details of the surface morphology, crystal structure, and chemical composition critically influence the photochemical and photocatalytic performance of the photocatalysts [270-274]. Therefore, these parameters need to be carefully controlled and evaluated when comparing photocatalytic activities of different materials.

\subsection{Chemical and Biological Sensing}

The unique optical properties of nanomaterials lend them conveniently for various optical sensing and detection of chemicals and biological samples. The optical luminescence from QDs is often used as a signature or probe [8, 97, 275-282]. For example, luminescent CdSe-ZnS core/shell quantum dot (QD) bioconjugates have been designed to detect proteolytic activity of enzymes by fluorescence resonance energy transfer (FRET) [283]. A modular peptide structure was developed for controlling the distance between the donors and acceptors. The high sensitivity of PL allows detection of single molecules and complex systems such as viruses [284].

\subsection{Photonics and Solid State Lighting}

Nanomaterials have found promising applications in photonics such as laser, LEDs, solid state lighting, and phosphors. In many of these applications, doping plays an important role, especially in phosphor materials. Lasers and LEDs based on thin films with thicknesses on the nanometer scale (2-D nanomaterials) have long been demonstrated and are 
currently used in commercial products. 1-D and 0-D nanomaterials have shown lasing properties based on optical pumping and demonstrated promising for technical applications [285, 286]. Lasing based on electrical pumping is a current challenge and if successful would represent a major technological breakthrough. The challenge is partly related to a higher density of surface trap states of 0-D and 1-D nanomaterials compared to their 2-D counter parts.

For laser applications, it is in principle possible to build lasers with different wavelengths by simply changing the particle size. There are two practical problems with this idea. First, the spectrum of most nanoparticles is usually quite broad due to homogeneous and inhomogeneous broadening. Second, the high density of trap states leads to fast relaxation of the excited charge carrier, making it difficult to create the population inversion necessary for lasing. When the surface of the particles is clean and has few defects, the idea of lasing can indeed be realized. This has been demonstrated mostly for nanoparticles self-assembled in clean environments based on physical methods, e.g. MBE (molecular beam epitaxy) [287-290] or MOCVD (metal organic chemical vapor deposition) [291]. Examples of quantum dot lasers include InGaAs [287], InAs [289], AlInAs [288, 290], and InP [292]. Stimulated emission has also been observed in GaN quantum dots by optical pumping [291]. The lasing action or stimulated emission has been observed mostly at low temperature [290, 292]. However, some room temperature lasing has also been achieved [287, 288].

Lasing action has been observed in colloidal nanoparticles of CdSe based on wet chemistry synthesis and optical pumping [286, 293]. It was found that, despite highly efficient intrinsic nonradiative Auger recombination, large optical gain can be developed at the wavelength of the emitting transition for close-packed solids of CdSe quantum dots. Narrow band stimulated emission with a pronounced gain threshold at wavelengths tunable with size of the nanocrystal was observed. This work demonstrates the feasibility of nanocrystal quantum dot lasers based on wet chemistry techniques. Whether real laser devices can be built based on these types of nanoparticles remains to be seen. Also, it is unclear if electrical pumping of such lasers can be realized. Likewise, nanoparticles can be potentially used for laser amplification and such an application has yet to be explored. Nanoparticles such as $\mathrm{TiO}_{2}$ have also been used to enhance stimulated emission for conjugated polymers based on multiple reflection effect [294].

Room temperature ultraviolet lasing in $\mathrm{ZnO}$ nanowire arrays has been demonstrated [285]. The $\mathrm{ZnO}$ nanowires grown on sapphire substrates were synthesized with a simple vapor transport and condensation process. The nanowires form a natural laser cavity with diameters varying from 20 to $150 \mathrm{~nm}$ and lengths up to $10 \mu \mathrm{m}$. Under optical excitation at $266 \mathrm{~nm}$, surface-emitting lasing action was observed at $385 \mathrm{~nm}$ with emission line width less than $0.3 \mathrm{~nm}$. Such miniaturized lasers could have many 
interesting applications ranging from optical storage to integrated optical communication devices.

Nanoparticles have been used for LED application in two ways. First, they are used to enhance light emission of LED devices with other materials, e.g. conjugated polymers, as the active media. The role of the nanoparticles, such as $\mathrm{TiO}_{2}$, is not completely clear but thought to enhance either charge injection or transport [295]. In some cases the presence of semiconductor nanocrystals in carrier-transporting polymers has been found to not only enhance the photoinduced charge generation efficiency but also extends the sensitivity range of the polymers, while the polymer matrix is responsible for charge transport [296]. This type of polymer/nanocrystal composite materials can have improved properties over the individual constituent components and may have interesting applications. Second, the nanoparticles are used as the active material for light generation directly [88, 297-299]. In this case, the electron and hole are injected directly into the CB and VB, respectively, of the NPs and the recombination of the electron and hole results in light emission. Several studies have been reported with the goal to optimize injection and charge transport in such device structures using CdS [299] and CdSe nanoparticles [297, 298].

Since the mobility of the charge carriers is usually much lower than in bulk single crystals, charge transport is one of the major limitations in efficient light generation in such devices. For example, photoconductivity and electric field induced photoluminescence quenching studies of closepacked CdSe quantum dot solids suggest that photoexcited, quantum confined excitons are ionized by the applied electric field with a rate dependent on both the size and surface passivation of the quantum dots $[300,301]$. Separation of electron-hole pairs confined to the core of the dot requires significantly more energy than separation of carriers trapped at the surface and occurs through tunneling processes. New nanostructures such nanowires [285, 302], nanorods [16, 72, 303, 304], and nanobelts [305], may provide some interesting alternatives with better transport properties than nanoparticles. Devices such as LEDs and solar cells based on such nanostructures are expected to be developed in the next few years.

Solid state lighting is an area of fast growing interest. Approximately $30 \%$ of the United States electricity is consumed by lighting, an industry that is largely dominated by relatively old technologies such as the incandescent and fluorescent light bulb. New innovations in lower cost and higher efficiency solid state lighting are expected to significantly reduce our dependence on fossil fuels. A solid state lighting technology that is already compatible with low cost manufacturing is AC powder electroluminescence (ACPEL). Discovered in 1936 [306], powder electroluminescence utilizes emission from $\sim 40-50$ micron sized doped$\mathrm{ZnS}: \mathrm{Cu}, \mathrm{Cl}$ phosphor particles and requires relatively low applied electric fields $\left(10^{4} \mathrm{~V} / \mathrm{cm}\right)$ compared to DC EL which requires electric fields near $10^{6} \mathrm{~V} / \mathrm{cm}$. More recently, microencapsulation technology has been 
successfully applied to $\mathrm{ZnS}: \mathrm{Cu}, \mathrm{Cl}$ powder phosphors so that the emissive particles can be deposited on plastic substrates under open air, non cleanroom conditions using low cost, large area print-based manufacturing. Consequently, ACPEL lights are one of the least expensive large area solid state lighting technologies, and the characteristic blue-green light can now be found in many products. Furthermore, fluorescent energy conversion and doping can readily be used to convert the blue-green light to the white light preferred for normal everyday lighting. Using a variety of other dopants (I, Br, Al, Mn, Pr, Tm etc.) other colors can also be obtained [307, 308].

The mechanism for light emission from $\mathrm{ZnS}: \mathrm{Cu}, \mathrm{Cl}$ particles is thought to be due to localized electron and hole injection near $\mathrm{Cu}_{\mathrm{x}} \mathrm{S}$ inclusions which requires an alternating (AC) field to enable the frequency-dependent electron-hole recombination. While this process is highly efficient, the lifetime and power efficiency of ACPEL lights are heretofore too low to provide a replacement for white lights. The power efficiency is limited by the large $\mathrm{ZnS}: \mathrm{Cu}, \mathrm{Cl}$ particle size $(>20 \mu \mathrm{m})$ over which the electric field is dropped, resulting in the need for higher voltages, $\sim 120 \mathrm{~V}$, to achieve the brightness needed for solid state lighting. Research focused on phosphor nanoparticles has attempted to address the voltage issue; however, these systems typically have significantly reduced lifetime and quantum efficiency due to poor charge transport and/or trapping. For example, an EXAFS study has been conducted to understand how the degradation depends on the local microstructure about $\mathrm{Cu}$ in doped- $\mathrm{ZnS}: \mathrm{Cu}, \mathrm{Cl}$ and have found that the degradation process is reversible through modest application of elevated temperatures $\left(\sim 200^{\circ} \mathrm{C}\right)$ [309]. Note that although the particle size is large (typically $20-50 \mu \mathrm{m}$ ), the active regions within each particle are near $\mathrm{Cu}_{\mathrm{x}} \mathrm{S}$ precipitates a few $\mathrm{nm}$ in size; hence it is essential to understand the $\mathrm{Cu}_{\mathrm{x}} \mathrm{S}$ nanoparticle precipitates within the $\mathrm{ZnS}$ host and how they interact with other optically active centers. Nanoparticle and nanorod systems exhibiting AC EL have been reported recently and will be studied for comparison [308, 310].

\subsection{Single Molecule and Single Nanoparticle Spectroscopy}

Most spectroscopy studies of nanoparticles have been carried out on ensembles of a large number of particles. The properties measured are thus ensemble averages of the properties of individual particles. Due to heterogeneous distributions in size, shape, environment, and surface properties, the spectrum measured is thus inhomogeneously broadened. This results in loss of spectral information. For instance, it has been predicted by theory that nanocrystallites should have a spectrum of discrete, atomic-like energy states [1,2]. However, transition line widths observed experimentally appear significantly broader than expected, even though the discrete nature of the excited states has been verified $[311,312]$. 
This is true even when size-selective optical techniques are used to extract homogeneous line widths [311-317].

One way to solve the above problem is to make particles with truly uniform size, surface, environment, and shape. However, this is almost impossible or at least very difficult, especially with regard to the surface. Another approach to remove the heterogeneity is to conduct the measurement on one single particle. This approach is similar to that used in the field of single-molecular spectroscopy [318, 319]. A number of single nanoparticle studies have been reported on semiconductor nanoparticles, including CdS [320] and CdSe [321-327]. Compared to ensemble averaged samples, single particle spectroscopy studies of CdSe nanoparticles revealed several new features, including fluorescence blinking, ultra narrow transition line width, a range of phonon couplings between individual particles, and spectral diffusion of the emission spectrum over a wide range of energies [327, 328].

One interesting observation in measuring the emission of a single nanoparticle is an intermittent on-off behavior in emission intensity under CW light excitation [323]. The intermittency observed was attributed to an Auger photoionization mechanism that leads to ejection of one charge (electron or hole) outside the particle. A "dark exciton" state was assigned to such ionized nanocrystal in which the emission is quenched because of excess charge. Similar studies on single $\mathrm{CdSe} / \mathrm{CdS}$ core/shell nanocrystals as a function of temperature and excitation intensity and the observations are consistent with a darkening mechanism that is a combination of Auger photoionization and thermal trapping of charge [329].

Interestingly, single particle spectroscopy also reveals non-linear optical properties of single nanoparticles. For instance, in the low temperature near-field absorption spectroscopy study of InGaAs single quantum dots, the absorption change was found to depend non-linearly on the excitation intensity [330]. This non-linearity was suggested to originate from state filling of the ground state.

Single nanoparticle spectroscopy should be a powerful technique for studying properties of doped semiconductor nanoparticles in terms of the uniformity of doping, location and state of the dopant ions, and interaction between the dopant and host. To date, only one report on doped semiconductor nanoparticles, Te-doped CdSe, has been reported [331]. The photoluminescence spectra from single Te-doped CdSe $(6 \mathrm{~nm}$ in diameter, passivated by $\mathrm{ZnS}$ ) measured at room temperature was analyzed in comparison with those from undoped CdSe. No difference was observed in the emission linewidths of individual particles of doped and undoped samples, though emissions from doped samples were found to have wider ensemble bands due to increased inhomogeneity because of doping. Several dopant emissions showed irreversible blue shifts with repeated measurements, which significantly exceeded those observed for the undoped ones. The shifts were attributed to instability of the dopant in a nanocrystal upon excitation. 


\section{Concluding Remarks}

While significant progress as been made in the last decade in the synthesis, characterization, and exploration of applications of nanomaterials, there is still much room for further study in all these areas. On the synthesis front, better control and assembly of nanostructures and functionality is important, including size, shape, surface, impurity, and interaction between structures. For better characterization, we need to have more and higher precision experimental tools for atomic level studies. Single particle studies represent a good step in this regard but still lack atomic resolution usually. In the ideal scenario, one would like to have high resolution in space, time, and energy that are atom or element specific. This is needed for a complete understanding of structural, optical, electronic, magnetic, and dynamic properties.

For applications, one key frontier issue is integration of different nanostructured components for desired functionality. This involves understanding, engineering, and controlling interfaces between the different components. Therefore, it is important to be able to design and fabricate interfaces with atomic precision since it is the atomic details at the interfaces that usually play a critical role in the properties and functionalities of the interface and assembled or integrated systems. This may require synergetic multi- and inter- disciplinary approaches involving collaborative and collective efforts from scientists and engineers in different areas and fields.

\section{Acknowledgements}

This work is partially supported by the BES Division of the US Department of Energy, National Science Foundation, NASA-UARC, UCMEXUS, and UCSC (JZZ). The work at Lawrence Livermore National Laboratory was performed under the auspices of the University of California under DOE contract No. W-7405-Eng-48 (CDG).

\section{References}

1. A.L. Efros and A.L. Efros, Fizika i Tekhnika Poluprovodnikov 16, 1209 (1982).

2. L.E. Brus, J. Chem. Phys. 80, 4403 (1984).

3. C.B. Murray, C.R. Kagan and M.G. Bawendi, Ann. Rev. Materials Science 30, 545 (2000).

4. N. Zaitseva, Z.R. Dai, F.R. Leon and D. Krol, J. Am. Chem. Soc. 127, 10221 (2005).

5. C. Burda, X.B. Chen, R. Narayanan and M.A. El-Sayed, Chem. Rev. 105, 1025 (2005).

6. J.Z. Zhang, Acc. Chem. Res. 30, 423 (1997). 
7. J.Z. Zhang, J. Phys. Chem. B 104, 7239 (2000).

8. C.B. Murray, D.J. Norris and M.G. Bawendi, J. Am. Chem. Soc. 115, 8706 (1993).

9. C.B. Murray, M. Nirmal, D.J. Norris and M.G. Bawendi, Z Phys D Atom Mol Cl 26, S231 (1993).

10. A.P. Alivisatos, Science 271, 933 (1996).

11. A.A. Guzelian, J.E.B. Katari, A.V. Kadavanich, U. Banin, K. Hamad, E. Juban, A.P. Alivisatos, R.H. Wolters, C.C. Arnold and J.R. Heath, J. Phys. Chem. 100, 7212 (1996).

12. X.G. Peng, M.C. Schlamp, A.V. Kadavanich and A.P. Alivisatos, J. Am. Chem. Soc. 119, 7019 (1997).

13. X.G. Peng, Chem-Eur J 8, 335 (2002).

14. J. Joo, H.B. Na, T. Yu, J.H. Yu, Y.W. Kim, F. Wu, J.Z. Zhang and T. Hyeon, J. Am. Chem. Soc. 125, 11100 (2003).

15. A.Y. Nazzal, X.Y. Wang, L.H. Qu, W. Yu, Y.J. Wang, X.G. Peng and M. Xiao, J. Phys. Chem. B 108, 5507 (2004).

16. L. Manna, E.C. Scher and A.P. Alivisatos, J. Am. Chem. Soc. 122, 12700 (2000).

17. J. Wang, M. Nozaki, M. Lachab, R.S.Q. Fareed, Y. Ishikawa, T. Wang, Y. Naoi and S. Sakai, J Cryst Growth 200, 85 (1999).

18. S.Y. Lu and S.W. Chen, J. Am. Ceram. Soc. 83, 709 (2000).

19. J.W. Wang, Y.P. Sun, Z.H. Liang, H.P. Xu, C.M. Fan and X.M. Chen, Rare Metal Mat Eng 33, 478 (2004).

20. Y.J. Li, P.B. Shi, R. Duan, B.R. Zhang, Y.P. Qiao, G.G. Qin and L. Huang, J. Infrared Millimeter Waves 23, 176 (2004).

21. M. Sacilotti, J. Decobert, H. Sik, G. Post, C. Dumas, P. Viste and G. Patriarche, J. Cryst. Growth 272, 198 (2004).

22. H.T. Lin, J.L. Huang, S.C. Wang and C.F. Lin, J. Alloy Compd. 417, 214 (2006).

23. D.Y. He, X.Q. Wang, Q. Chen, J.S. Li, M. Yin, A.V. Karabutov and A.G. Kazanskii, Chinese Sci. Bull. 51, 510 (2006).

24. Z.Z. Ye, J.Y. Huang, W.Z. Xu, J. Zhou and Z.L. Wang, Solid State Commun. 141, 464 (2007).

25. J.Y. Wang and T. Ito, Diamond Related Materials 16, 589 (2007).

26. R.L. Barns and W.C. Ellis, J. Appl. Phys. 36, 2296 (1965).

27. R.S. Wagner and W.C. Ellis, Transa. Metallurgical Soc. Aime 233, 1053 (1965).

28. J.P. Sitarik and W.C. Ellis, J. Appl. Phys. 37, 2399 (1966).

29. R.S. Wagner and C.J. Doherty, J. Electrochem. Soc. 115, 93 (1968).

30. P.X. Gao and Z.L. Wang, J. Phys. Chem. B 108, 7534 (2004).

31. Y. Ding, P.X. Gao and Z.L. Wang, J. Am. Chem. Soc. 126, 2066 (2004).

32. F.M. Kolb, H. Hofmeister, R. Scholz, M. Zacharias, U. Gosele, D.D. Ma and S.T. Lee, J. Electrochem. Soc. 151, G472 (2004).

33. M.C. Yang, J. Shieh, T.S. Ko, H.L. Chen and T.C. Chu, Jpn. J. Appl. Phys. 1 44, 5791 (2005). 
34. J.L. Taraci, T. Clement, J.W. Dailey, J. Drucker and S.T. Picraux, Nuclear Instru. Meth. Phys. Res. B 242, 205 (2006).

35. A.M. Morales and C.M. Lieber, Science 279, 208 (1998).

36. Z.W. Pan, Z.R. Dai, C. Ma and Z.L. Wang, J. Am. Chem. Soc. 124, 1817 (2002).

37. J.R. Morber, Y. Ding, M.S. Haluska, Y. Li, P. Liu, Z.L. Wang and R.L. Snyder, J. Phys. Chem. B 110, 21672 (2006).

38. X.F. Duan and C.M. Lieber, J. Am. Chem. Soc. 122, 188 (2000).

39. Y.Q. Chen, K. Zhang, B. Miao, B. Wang and J.G. Hou, Chem. Phys. Lett. 358, 396 (2002).

40. J.C. Johnson, H.J. Choi, K.P. Knutsen, R.D. Schaller, P.D. Yang and R.J. Saykally, Nat. Mater. 1, 106 (2002).

41. C.Y. Meng, B.L. Shih and S.C. Lee, J. Nanoparticle Research 9, 657 (2007).

42. M. Gentili, C. Giovannella and S. Selci, eds. Nanolithography: A Borderland between STM, EB, IB, and X-Ray Lithographies, Kluwer Academic Publishers, New York, 1994.

43. J.Z. Zhang, Z.L. Wang, J. Liu, S. Chen and G.-Y. Liu, Selfassembled Nanostructures. Nanoscale Science and Technology, New York, Kluwer Academic/Plenum Publishers, 2003.

44. G. Cao, Nanostructures \& Nanomaterials: Synthesis, Properties \& Applications, London, Imperial College Press, 2004.

45. W. Chen, J.Z. Zhang and A. Joly, J. Nanosci.Nanotech. 4, 919 (2004).

46. J.D. Bryan and D.R. Gamelin, Prog. Inorg. Chem. 54, 47 (2005).

47. G.Y. Liu, S. Xu and Y.L. Qian, Acc. Chem. Res. 33, 457 (2000).

48. Z.L. Wang, J. Phys. Chem. B 104, 1153 (2000).

49. Z.L. Wang, P. Poncharal and W.A. de Heer, Microscopy and Microanalysis 6, 224 (2000).

50. R.L. Whetten, J.T. Khoury, M.M. Alvarez, S. Murthy, I. Vezmar, Z.L. Wang, P.W. Stephens, C.L. Cleveland, W.D. Luedtke and U. Landman, Advan. Mater. 8, 428 (1996).

51. C.B. Murray, C.R. Kagan and M.G. Bawendi, Science 270, 1335 (1995).

52. G. Binnig, C.F. Quate and C. Gerber, Phys. Rev. Lett. 56, 930 (1986).

53. G. Binnig, H. Rohrer, C. Gerber and E. Weibel, Appl. Phys. Lett. 40, 178 (1982).

54. H.J. Guntherodt, D. Anselmetti and E. Meyer, eds. Forces in scanning probe methods, NATO ASI Series. Series E, Applied sciences. Vol. 286, Kluwer Academic, 1995.

55. J.I. Goldstein, D.E. Newbury, P. Echlin, D.C. Joy, J. Romig, A.D., C.E. Lyman, C. Fiori and E. Lifshin, Scanning Electron Microscopy and X-ray Microanalysis. 2nd ed, New York, Plenum Press, 1992.

56. Z.L. Wang, ed. Characterization of Nanophase Materials. WileyVCH, New York, 2000.

57. Z.L. Wang, Advan. Mater. 10, 13 (1998). 
58. T.L. Barr, Modern ESCA: The Principles and Practice of X-Ray Photoelectron Spectroscopy, New York, CRC Press, 1994.

59. D.C. Koningsberger and R. Prins, eds. X-Ray Absorption: Principles, Applications, Techniques of EXAFS, SEXAFS and XANES, WileyInterscience, New York, 1988.

60. F. Wu, J.Z. Zhang, R. Kho and R.K. Mehra, Chem. Phys. Lett. 330, 237 (2000).

61. H. Dollefeld, H. Weller and A. Eychmuller, Nano Letters 1, 267 (2001).

62. A.M. Malvezzi, M. Patrini, A. Stella, P. Tognini, P. Cheyssac and R. Kofman, Eur. Phys. J. D 16, 321 (2001).

63. A. Stella, S. Achilli, M. Allione, A.M. Malvezzi, M. Patrini and R. Kofman, Microelectron J. 34, 619 (2003).

64. Z. Gui, X. Wang, J. Liu, S.S. Yan, Y.Y. Ding, Z.Z. Wang and Y. Hu, J. Solid State Chem. 179, 1984 (2006).

65. C.Y. Wang, H. Groenzin and M.J. Shultz, Langmuir 19, 7330 (2003).

66. C.Y. Wang, H. Groenzin and M.J. Shultz, J. Phys. Chem. B 108, 265 (2004).

67. K.C. Jena, P.B. Bisht, M.M. Shaijumon and S. Ramaprabhu, Opt Commun. 273, 153 (2007).

68. M. Gratzel, Heterogeneous Photochemical Electron Transfer, Boca Raton, CRC Press, 1989.

69. V.L. Colvin, A.N. Goldstein and A.P. Alivisatos, J. Am. Chem. Soc. 114, 5221 (1992).

70. D. Duonghong, J.J. Ramsden and M. Gratzel, J. Am. Chem. Soc. 104, 2977 (1982).

71. J.Z. Zhang, R.H. O'Neil and T.W. Roberti, J. Phys. Chem. 98, 3859 (1994).

72. Z. Adam and X. Peng, J. Am. Chem. Soc. 123, 183 (2001).

73. A. Wolcott, D. Gerion, M. Visconte, J. Sun, A. Schwartzberg, S.W. Chen and J.Z. Zhang, J. Phys. Chem. B 110, 5779 (2006).

74. R.A. Bley, S.M. Kauzlarich, J.E. Davis and H.W.H. Lee, Chem. Mater. 8, 1881 (1996).

75. T. Takagahara, Phys. Rev. B. 36, 9293 (1987).

76. Y. Kayanuma, Phys. Rev. B. 38, 9797 (1988).

77. W.W. Yu, L.H. Qu, W.Z. Guo and X.G. Peng, Chem. Mater. 15, 2854 (2003).

78. B. Valeur, Molecular Fluorescence: Principles and Applications, New York, Wiley-VCH, 2001.

79. L.R. Becerra, C.B. Murray, R.G. Griffin and M.G. Bawendi, J. Chem. Phys. 100, 3297 (1994).

80. J.E.B. Katari, V.L. Colvin and A.P. Alivisatos, J. Phys. Chem. 98, 4109 (1994).

81. L. Spanhel, M. Haase, H. Weller and A. Henglein, J. Am. Chem. Soc. 109, 5649 (1987).

82. P.V. Kamat and N.M. Dimitrijevic, J. Phys. Chem. 93, 4259 (1989). 
83. P.V. Kamat, M.D. Vanwijngaarden and S. Hotchandani, Israel J. Chem. 33, 47 (1993).

84. C. Luangdilok and D. Meisel, Israel J. Chem. 33, 53 (1993).

85. M.Y. Gao, S. Kirstein, H. Mohwald, A.L. Rogach, A. Kornowski, A. Eychmuller and H. Weller, J. Phys. Chem. B 102, 8360 (1998).

86. T.W. Roberti, N.J. Cherepy and J.Z. Zhang, J. Chem. Phys. 108, 2143 (1998).

87. M.A. Hines and P. Guyot-Sionnest, J. Phys. Chem. 100, 468 (1996).

88. M.C. Schlamp, X.G. Peng and A.P. Alivisatos, J. Appl. Phys. 82, 5837 (1997).

89. P. Palinginis and W. Hailin, Appl. Phys. Lett. 78, 1541 (2001).

90. M.A. Hines and P. Guyot-Sionnest, J. Phys. Chem. B 102, 3655 (1998).

91. A.A. Patel, F.X. Wu, J.Z. Zhang, C.L. Torres-Martinez, R.K. Mehra, Y. Yang and S.H. Risbud, J. Phys. Chem. B 104, 11598 (2000).

92. A. Sengupta, B. Jiang, K.C. Mandal and J.Z. Zhang, J. Phys. Chem. B 103, 3128 (1999).

93. M.C. Brelle, C.L. Torres-Martinez, J.C. McNulty, R.K. Mehra and J.Z. Zhang, Pure Appl. Chem. 72, 101 (2000).

94. M.C. Brelle, J.Z. Zhang, L. Nguyen and R.K. Mehra, J. Phys. Chem. A 103, 10194 (1999).

95. S.R. Cordero, P.J. Carson, R.A. Estabrook, G.F. Strouse and S.K. Buratto, J. Phys. Chem. B 104, 12137 (2000).

96. X.H. Gao, W.C.W. Chan and S.M. Nie, J. Biomed. Optics 7, 532 (2002).

97. D. Gerion, F. Pinaud, S.C. Williams, W.J. Parak, D. Zanchet, S. Weiss and A.P. Alivisatos, J. Phys. Chem. B 105, 8861 (2001).

98. X.H. Gao and S.M. Nie, Analyt. Chem. 76, 2406 (2004).

99. N.M. Dimitrijevic and P.V. Kamat, J. Phys. Chem. 91, 2096 (1987).

100. Y. Wang, A. Suna, J. McHugh, E.F. Hilinski, P.A. Lucas and R.D. Johnson, J. Chem. Phys. 92, 6927 (1990).

101. T. Vossmeyer, L. Katsikas, M. Giersig, I.G. Popovic, K. Diesner, A. Chemseddine, A. Eychmuller and H. Weller, J. Phys. Chem. 98, 7665 (1994).

102. A. Henglein, A. Kumar, E. Janata and H. Weller, Chem. Phys. Lett. 132, 133 (1986).

103. K.I. Kang, A.D. Kepner, S.V. Gaponenko, S.W. Koch, Y.Z. Hu and N. Peyghambarian, Phys. Rev. B 48, 15449 (1993).

104. V. Klimov, S. Hunsche and H. Kurz, Phys. Rev. B 50, 8110 (1994).

105. V. Dneprovskii, N. Gushina, O. Pavlov, V. Poborchii, I. Salamatina and E. Zhukov, Physics Letters A 204, 59 (1995).

106. N.V. Gushchina, V.S. Dneprovskii, E.A. Zhukov, O.V. Pavlov, V.V. Poborchii and I.A. Salamatina, Jetp. Lett.-Engl. Tr. 61, 507 (1995).

107. V. Dneprovskii, A. Eev, N. Gushina, D. Okorokov, V. Panov, V. Karavanskii, A. Maslov, V. Sokolov and E. Dovidenko, Phys. Status Solidi B 188, 297 (1995). 
108. V. Dneprovskii, N. Gushina, D. Okorokov, V. Karavanskii and E. Dovidenko, Superlattice Microst. 17, 41 (1995).

109. F.Z. Henari, K. Morgenstern, W.J. Blau, V.A. Karavanskii and V.S. Dneprovskii, Appl. Phys. Lett. 67, 323 (1995).

110. B.L. Yu, C.S. Zhu and F.X. Gan, J. Appl. Phys. 82, 4532 (1997).

111. Y. Wang, Acc. Chem. Res. 24, 133 (1991).

112. T. Dannhauser, M. O'Neil, K. Johanseon, D. Whitter and G. McLendon, J. Phys. Chem. 90, 6074 (1986).

113. X.C. Ai, L. Guo, Y.H. Zou, Q.S. Li and H.S. Zhu, Mater. Lett. 38, 131 (1999).

114. Y.R. Shen, The Principles of Nonlinear Optics, New York, J. Wiley, 1984.

115. T. Yamaki, K. Asai, K. Ishigure, K. Sano and K. Ema, Synthet. Metal 103, 2690 (1999).

116. M. Jacobsohn and U. Banin, J. Phys. Chem. B 104, 1 (2000).

117. Z. Yu, F. Dgang, W. Xin, L. Juzheng and L. Zuhong, Colloids Surfaces A 181, 145 (2001).

118. J. Lenglet, A. Bourdon, J.C. Bacri, R. Perzynski and G. Demouchy, Phys. Rev. B 53, 14941 (1996).

119. T. Takagahara, Solid State Commun. 78, 279 (1991).

120. T. Takagahara, Surface Sci. 267, 310 (1992).

121. Y. Kayanuma, J. Phys. Soc. Japan 62, 346 (1993).

122. B. Clerjaud, F. Gendron and C. Porte, Appl. Phys. Lett. 38, 212 (1981).

123. Y. Mita, in Phosphor Handbook, S.a.Y. Shionoya, W.M., Editor, CRC Press, New York, 1999.

124. L.G. Quagliano and H. Nather, Appl. Phys. Lett. 45, 555 (1984).

125. E.J. Johnson, J. Kafalas, R.W. Davies and W.A. Dyes, Appl. Phys. Lett. 40, 993 (1982).

126. E. Poles, D.C. Selmarten, O.I. Micic and A.J. Nozik, Appl. Phys. Lett. 75, 971 (1999).

127. Y.H. Cho, D.S. Kim, B.D. Choe, H. Lim, J.I. Lee and D. Kim, Phys. Rev. B 56, R4375 (1997).

128. M. Potemski, R. Stepniewski, J.C. Maan, G. Martinez, P. Wyder and B. Etienne, Phys. Rev. Lett. 66, 2239 (1991).

129. P. Vagos, P. Boucaud, F.H. Julien, J.M. Lourtioz and R. Planel, Phys. Rev. Lett. 70, 1018 (1993).

130. W. Seidel, A. Titkov, J.P. Andre, P. Voisin and M. Voos, Phys, Rev, Lett, 73, 2356 (1994).

131. F. Driessen, H.M. Cheong, A. Mascarenhas, S.K. Deb, P.R. Hageman, G.J. Bauhuis and L.J. Giling, Phys. Rev. B 54, R5263 (1996).

132. R. Hellmann, A. Euteneuer, S.G. Hense, J. Feldmann, P. Thomas, E.O. Gobel, D.R. Yakovlev, A. Waag and G. Landwehr, Phys. Rev. B 51, 18053 (1995). 
133. Z.P. Su, K.L. Teo, P.Y. Yu and K. Uchida, Solid State Commun. 99, 933 (1996).

134. J. Zeman, G. Martinez, P.Y. Yu and K. Uchida, Phys. Rev. B 55, 13428 (1997).

135. L. Schrottke, H.T. Grahn and K. Fujiwara, Phys. Rev. B 56, 15553 (1997).

136. H.M. Cheong, B. Fluegel, M.C. Hanna and A. Mascarenhas, Phys. Rev. B 58, R4254 (1998).

137. Z. Chine, B. Piriou, M. Oueslati, T. Boufaden and B. El Jani, J. Luminescence 82, 81 (1999).

138. T. Kita, T. Nishino, C. Geng, F. Scholz and H. Schweizer, Phys. Rev. B 59, 15358 (1999).

139. S.C. Hohng and D.S. Kim, Appl. Phys. Lett. 75, 3620 (1999).

140. L. Schrottke, R. Hey and H.T. Grahn, Phys. Rev. B 60, 16635 (1999).

141. W. Heimbrodt, M. Happ and F. Henneberger, Phys. Rev. B 60, R16326 (1999).

142. T. Kita, T. Nishino, C. Geng, F. Scholz and H. Schweizer, J. Luminescence 87-9, 269 (2000).

143. A. Satake, Y. Masumoto, T. Miyajima, T. Asatsuma and T. Hino, Phys. Rev. B 61, 12654 (2000).

144. G.G. Zegrya and V.A. Kharchenko, Zhurnal Eksperimentalnoi I Teoreticheskoi Fiziki 101, 327 (1992).

145. S.A. Blanton, M.A. Hines, M.E. Schmidt and P. Guyotsionnest, J. Luminescence 70, 253 (1996).

146. I.V. Ignatiev, I.E. Kozin, H.W. Ren, S. Sugou and Y. Matsumoto, Phys. Rev. B 60, R14001 (1999).

147. P.P. Paskov, P.O. Holtz, B. Monemar, J.M. Garcia, W.V. Schoenfeld and P.M. Petroff, Appl. Phys. Lett. 77, 812 (2000).

148. H.X. Zhang, C.H. Kam, Y. Zhou, X.Q. Han, S. Buddhudu and Y.L. Lam, Opt. Mater. 15, 47 (2000).

149. W. Chen, A.G. Joly and J.Z. Zhang, Phys Rev B 6404, 1202 (2001).

150. A.G. Joly, W. Chen, J. Roark and J.Z. Zhang, J. Nanosci. Nanotech. 1, 295 (2001).

151. J.J. Shiang, A.N. Goldstein and A.P. Alivisatos, J. Chem. Phys. 92, 3232 (1990).

152. G. Scamarcio, M. Lugara and D. Manno, Phys. Rev. B 45, 13792 (1992).

153. J.J. Shiang, S.H. Risbud and A.P. Alivisatos, J. Chem. Phys. 98, 8432 (1993).

154. M. Abdulkhadar and B. Thomas, Nanostruct. Mater. 5, 289 (1995).

155. A.A. Sirenko, V.I. Belitsky, T. Ruf, M. Cardona, A.I. Ekimov and C. TralleroGiner, Phys. Rev. B 58, 2077 (1998).

156. V.G. Melehin and V.D. Petrikov, Physics of Low-Dimensional Structures 9-10, 73 (1999).

157. A.P. Alivisatos, T.D. Harris, P.J. Carroll, M.L. Steigerwald and L.E. Brus, J. Chem. Phys. 90, 3463 (1989). 
158. J.J. Shiang, I.M. Craig and A.P. Alivisatos, Z. Phys. D Atom Mol. Cl. 26, 358 (1993).

159. V. Spagnolo, G. Scamarcio, M. Lugara and G.C. Righini, Superlattice Microst. 16, 51 (1994).

160. J.J. Shiang, R.H. Wolters and J.R. Heath, J. Chem. Phys. 106, 8981 (1997).

161. V.A. Volodin, M.D. Efremov, V.A. Gritsenko and S.A. Kochubei, Appl. Phys. Lett. 73, 1212 (1998).

162. M.D. Efremov, V.V. Bolotov, V.A. Volodin and S.A. Kochubei, Solid State Commun. 108, 645 (1998).

163. W.F.A. Besling, A. Goossens and J. Schoonman, J. Phys. IV. 9, 545 (1999).

164. G.H. Li, K. Ding, Y. Chen, H.X. Han and Z.P. Wang, J. Appl. Phys. 88,1439 (2000).

165. J.R. Heath, J.J. Shiang and A.P. Alivisatos, J. Chem. Phys. 101, 1607 (1994).

166. Y.Y. Wang, Y.H. Yang, Y.P. Guo, J.S. Yue and R.J. Gan, Mater. Lett. 29, 159 (1996).

167. W.K. Choi, V. Ng, S.P. Ng, H.H. Thio, Z.X. Shen and W.S. Li, J. Appl. Phys. 86, 1398 (1999).

168. A.V. Kolobov, Y. Maeda and K. Tanaka, J. Appl. Phys. 88, 3285 (2000).

169. Y.W. Ho, V. Ng, W.K. Choi, S.P. Ng, T. Osipowicz, H.L. Seng, W.W. Tjui and K. Li, Scripta Materialia 44, 1291 (2001).

170. U. Pal and J.G. Serrano, Appl. Surf. Sci. 246, 23 (2005).

171. Y. Batra, D. Kabiraj and D. Kanjilal, Eur. Phys. J.-Appl. Phys. 38, 27 (2007).

172. R. Rinaldi, R. Cingolani, M. Ferrara, A.C. Maciel, J. Ryan, U. Marti, D. Martin, F. Moriergemoud and F.K. Reinhart, Appl. Phys. Lett. 64, 3587 (1994).

173. B. Schreder, A. Materny, W. Kiefer, G. Bacher, A. Forchel and G. Landwehr, J. Raman Spectroscopy 31, 959 (2000).

174. Y. Bae, N. Myung and A.J. Bard, Nano Letters 4, 1153 (2004).

175. Z.P. Wang, J. Li, B. Liu, J.Q. Hu, X. Yao and J.H. Li, J. Phys. Chem. B 109, 23304 (2005).

176. R.K. Ligman, L. Mangolini, U.R. Kortshagen and S.A. Campbell, Appl. Phys. Lett. 90, 061116 (2007).

177. C.Y. Lee, Y.T. Haung, W.F. Su and C.F. Lin, Appl. Phys. Lett. 89, 231116 (2006).

178. Y. Xuan, D.C. Pan, N. Zhao, X.L. Ji and D.G. Ma, Nanotechnology 17, 4966 (2006).

179. D.F. Underwood, T. Kippeny and S.J. Rosenthal, J. Phys. Chem. B 105, 436 (2001).

180. J.J. Cavaleri, D.E. Skinner, D.P. Colombo and R.M. Bowman, J. Chem. Phys. 103, 5378 (1995). 
181. D.E. Skinner, D.P. Colombo, J.J. Cavaleri and R.M. Bowman, J. Phys. Chem. 99, 7853 (1995).

182. V.I. Klimov, A.A. Mikhailovsky, D.W. McBranch, C.A. Leatherdale and M.G. Bawendi, Science 287, 1011 (2000).

183. F.X. Wu, J.H. Yu, J. Joo, T. Hyeon and J.Z. Zhang, Opt. Mater. 29, 858 (2007).

184. J.M. Luther, M.C. Beard, Q. Song, M. Law, R.J. Ellingson and A.J. Nozik, Nano Letters 7, 1779 (2007).

185. A. Luque, A. Marti and A.J. Nozik, Mrs Bulletin 32, 236 (2007).

186. G. Allan and C. Delerue, Phys. Rev. B 73, 205423 (2006).

187. J.E. Murphy, M.C. Beard, A.G. Norman, S.P. Ahrenkiel, J.C. Johnson, P.R. Yu, O.I. Micic, R.J. Ellingson and A.J. Nozik, J. Am. Chem. Soc. 128, 3241 (2006).

188. R.J. Ellingson, M.C. Beard, J.C. Johnson, P.R. Yu, O.I. Micic, A.J. Nozik, A. Shabaev and A.L. Efros, Nano Letters 5, 865 (2005).

189. V.I. Klimov, J. Phys. Chem. B 110, 16827 (2006).

190. B. Oregan and M. Gratzel, Nature 353, 737 (1991).

191. N.J. Cherepy, G.P. Smestad, M. Gratzel and J.Z. Zhang, J. Phys. Chem. B 101, 9342 (1997).

192. O. Khaselev and J.A. Turner, Science 280, 425 (1998).

193. J.A. Turner, Science 285, 687 (1999).

194. C.A. Parsons, M.W. Peterson, B.R. Thacker, J.A. Turner and A.J. Nozik, J. Phys. Chem. 94, 3381 (1990).

195. M. Gratzel, J. Photochem. Photobiol. C Photochem. Rev. 4, 145 (2003).

196. N.A. Anderson and T. Lian, Ann. Rev. Phys. Chem. 56, 491 (2005).

197. S. Logunov, T. Green, S. Marguet and M.A. El-Sayed, J. Phys. Chem. A 102, 5652 (1998).

198. C. Burda, T.C. Green, S. Link and M.A. El-Sayed, J. Phys. Chem. B 103, 1783 (1999).

199. W.Q. Peng, S.C. Qu, G.W. Cong, X.Q. Zhang and Z.G. Wang, J. Cryst. Growth 282, 179 (2005).

200. N. Pradhan and X.G. Peng, J. Am. Chem. Soc. 129, 3339 (2007).

201. G. Counio, T. Gacoin and J.P. Boilot, J. Phys. Chem. B 102, 5257 (1998).

202. J.Q. Yu, H.M. Liu, Y.Y. Wang, F.E. Fernandez and W.Y. Jia, J. Luminescence 76-7, 252 (1998).

203. A.D. Dinsmore, D.S. Hsu, H.F. Gray, S.B. Qadri, Y. Tian and B.R. Ratna, Appl. Phys. Lett. 75, 802 (1999).

204. W. Chen, R. Sammynaiken and Y.N. Huang, J. Appl. Phys. 88, 5188 (2000).

205. W. Chen, A.G. Joly and J.Z. Zhang, Phys. Rev. B 64, 41202 (2001).

206. M. Konishi, T. Isobe and M. Senna, J. Luminescence 93, 1 (2001).

207. K. Yan, C.K. Duan, Y. Ma, S.D. Xia and J.C. Krupa, Phys. Rev. B 58, 13585 (1998). 
208. T.J. Norman, D. Magana, T. Wilson, C. Burns, J.Z. Zhang, D. Cao and F. Bridges, J. Phys. Chem. B 107, 6309 (2003).

209. A.C. Tuan, J.D. Bryan, A.B. Pakhomov, V. Shutthanandan, S. Thevuthasan, D.E. McCready, D. Gaspar, M.H. Engelhard, J.W. Rogers, K. Krishnan, D.R. Gamelin and S.A. Chambers, Phys. Rev. B 70, 054424 (2004).

210. P.V. Radovanovic, N.S. Norberg, K.E. McNally and D.R. Gamelin, J. Am. Chem. Soc. 124, 15192 (2002).

211. R. Viswanatha, S. Sapra, S. Sen Gupta, B. Satpati, P.V. Satyam, B.N. Dev and D.D. Sarma, J. Phys. Chem. B 108, 6303 (2004).

212. R. Viswanatha, S. Chakraborty, S. Basu and D.D. Sarma, J. Phys. Chem. B 110, 22310 (2006).

213. R. Asahi, T. Morikawa, T. Ohwaki, K. Aoki and Y. Taga, Science 293, 269 (2001).

214. C. Burda, Y.B. Lou, X.B. Chen, A.C.S. Samia, J. Stout and J.L. Gole, Nano Letters 3, 1049 (2003).

215. L.H. Huang, Z.X. Sun and Y.L. Liu, J. Ceramic Soc. Japan 115, 28 (2007).

216. J.W. Wang, W. Zhu, Y.Q. Zhang and S.X. Liu, J. Phys. Chem. C 111, 1010 (2007).

217. M. Sathish, B. Viswanathan, R.P. Viswanath and C.S. Gopinath, Chem. Mater. 17, 6349 (2005).

218. S. Moribe, T. Ikoma, K. Akiyama, Q.W. Zhang, F. Saito and S. Tero-Kubota, Chem. Phys. Lett. 436, 373 (2007).

219. H.Y. Chen, A. Nambu, W. Wen, J. Graciani, Z. Zhong, J.C. Hanson, E. Fujita and J.A. Rodriguez, J.Phys. Chem. C 111, 1366 (2007).

220. S. Yin, Y. Aita, M. Komatsu and T. Sato, J. European Ceramic Soc. 26, 2735 (2006).

221. R.N. Bhargava, D. Gallagher, X. Hong and A. Nurmikko, Phys. Rev. Lett. 72, 416 (1994).

222. R.N. Bhargava, D. Gallagher and T. Welker, J.Luminescence 60-1, 275 (1994).

223. R.N. Bhargava, J. Luminescence 70, 85 (1996).

224. H.E. Gumlich, J. Luminescence 23, 73 (1981).

225. A.A. Bol and A. Meijerink, Phys. Rev. B 58, R15997 (1998).

226. N. Murase, R. Jagannathan, Y. Kanematsu, M. Watanabe, A. Kurita, K. Hirata, T. Yazawa and T. Kushida, J. Phys. Chem. B 103, 754 (1999).

227. B.A. Smith, J.Z. Zhang, A. Joly and J. Liu, Phys. Rev. B 62, 2021 (2000).

228. J.H. Chung, C.S. Ah and D.-J. Jang, J. Phys. Chem. B 105, 4128 (2001).

229. T. Toyama, D. Adachi, M. Fujii, Y. Nakano and H. Okamoto, J. Non-Crystalline Solids 299, 1111 (2002).

230. E.M. Olano, C.D. Grant, T.J. Norman, E.W. Castner and J.Z. Zhang, J. Nanosci. Nanotech. 5, 1492 (2005). 
231. A. Wolcott, T.R. Kuykendall, W. Chen, S.W. Chen and J.Z. Zhang, J. Phys. Chem. B 110, 25288 (2006).

232. S. Uchida, R. Chiba, M. Tomiha, N. Masaki and M. Shirai, Electrochem. 70, 418 (2002).

233. W.U. Huynh, J.J. Dittmer, W.C. Libby, G.L. Whiting and A.P. Alivisatos, Adv. Funct. Mater. 13, 73 (2003).

234. P. Gould, Materials Today 9, 18 (2006).

235. M. Law, L.E. Greene, A. Radenovic, T. Kuykendall, J. Liphardt and P.D. Yang, J. Phys. Chem. B 110, 22652 (2006).

236. K.S. Kim, Y.S. Kang, J.H. Lee, Y.J. Shin, N.G. Park, K.S. Ryu and S.H. Chang, B Kor. Chem. Soc. 27, 295 (2006).

237. Y.B. Liu, B.X. Zhou, B.T. Xiong, J. Bai and L.H. Li, Chinese Sci. Bull. 52, 1585 (2007).

238. T.Y. Lee, P.S. Alegaonkar and J.B. Yoo, Thin Solid Films 515, 5131 (2007).

239. E. Joanni, R. Savu, M.D. Goes, P.R. Bueno, J.N. de Freitas, A.F. Nogueira, E. Longo and J.A. Varela, Scripta Materialia 57, 277 (2007).

240. K. Robbie, J.C. Sit and M.J. Brett, J. Vacuum Science Technology B 16, 1115 (1998).

241. Y.P. Zhao, D.X. Ye, G.C. Wang and T.M. Lu, Nano Letters 2, 351 (2002).

242. W.W. Dunn, Y. Aikawa and BardmA.J., J. Am. Chem. Soc. 6893 (1981).

243. H. Harada, T. Ueda and T. Sakata, J. Phys. Chem. 93, 1542 (1989).

244. L.F. Lin and R.R. Kuntz, Langmuir 8, 870 (1992).

245. P. Zuman and Z. Fijalek, J. Electroanal. Chem. Interfacial Electrochem. 296, 583 (1990).

246. H.Y. Wang, R.E. Partch and Y.Z. Li, J. Org. Chem. 62, 5222 (1997).

247. I. Willner, F. Patolsky and J. Wasserman, Angew. Chem. Int. Edit 40, 1861 (2001).

248. C. Nasr, P.V. Kamat and S. Hotchandani, J. Electroanal. Chem. 420, 201 (1997).

249. S.G. Hickey, D.J. Riley and E.J. Tull, J. Phys. Chem. B 104, 7623 (2000).

250. G. Hodes, Israel Journal of Chemistry 33, 95 (1993).

251. K. Keis, L. Vayssieres, H. Rensmo, S.E. Lindquist and A. Hagfeldt, J. Electrochem. Soc.y 148, A149 (2001).

252. L. Zhang, M.Z. Yang, E.Q. Gao, X.B. Qiao, Y.Z. Hao, Y.Q. Wang, S.M. Cai, F.S. Meng and H. Tian, Chem. J. Chinese UniversitiesChinese 21, 1543 (2000).

253. Y. Ren, Z. Zhang, E. Gao, S. Fang and S. Cai, J. Appl. Electrochem. 31, 445 (2001).

254. X.M. Qian, D.Q. Qin, Q. Song, Y.B. Bai, T.J. Li, X.Y. Tang, E.K. Wang and S.J. Dong, Thin Solid Films 385, 152 (2001). 
255. M.S. Liu, M.Z. Yang, Y.Z. Hao, S.M. Cai and Y.F. Li, Acta Chim. Sinica 59, 377 (2001).

256. T. Yoshida, K. Terada, D. Schlettwein, T. Oekermann, T. Sugiura and H. Minoura, Advan. Mater. 12, 1214 (2000).

257. J.F. Suyver, R. Bakker, A. Meijerink and J.J. Kelly, Phys. Status Solidi B 224, 307 (2001).

258. C. Santato, M. Ulmann and J. Augustynski, J. Phys. Chem. B 105, 936 (2001).

259. K. Vinodgopal, I. Bedja and P.V. Kamat, Chem. Mater. 8, 2180 (1996).

260. S.K. Poznyak, D.V. Talapin and A.I. Kulak, J. Phys. Chem. B 105, 4816 (2001).

261. M.A. Fox and M.T. Dulay, Chem. Rev. 93, 341 (1993).

262. W.Y. Choi, A. Termin and M.R. Hoffmann, J. Phys. Chem. 98, 13669 (1994).

263. W.Y. Choi, A. Termin and M.R. Hoffmann, Angew. Chem., Int. Ed. Engl. 33, 1091 (1994).

264. M.R. Hoffmann, S.T. Martin, W.Y. Choi and D.W. Bahnemann, Chem. Rev. 95, 69 (1995).

265. L.Q. Jing, X.J. Sun, J. Shang, W.M. Cai, Z.L. Xu, Y.G. Du and H.G. Fu, Sol, Energ, Mat, Sol, Cell 79, 133 (2003).

266. D.F. Ollis and H. Al-Ekabi, Photocatalytic purification and treatment of water and air : proceedings of the 1st International Conference on $\mathrm{TiO}_{2}$ Photocatalytic Purification and Treatment of Water and Air, London, Ontario, Canada, 8-13 November, 1992. Trace metals in the environment ; 3, Amsterdam, New York, Elsevier, 1993.

267. A. Mills, R.H. Davies and D. Worsley, Chem. Soc. Rev. 22, 417 (1993).

268. N. Serpone and R.F. Khairutdinov, in Semiconductor NanoclustersPhysical, Chemical, and Catalytic Aspects, P.V. Kamat and D. Meisel, Editors, Elsevier, New York, 1997.

269. K. Okamoto, Y. Yamamoto, H. Tanaka, M. Tanaka and A. Itaya, B. Chem. Soc. Japan 58, 2015 (1985).

270. C.H. Kwon, H.M. Shin, J.H. Kim, W.S. Choi and K.H. Yoon, Mate.r Chem. Phys. 86, 78 (2004).

271. Y. Nemoto and T. Hirai, B. Chem. Soc. Jpn. 77, 1033 (2004).

272. Y. Jiang, P. Zhang, Z.W. Liu and F. Xu, Mater. Chem. Phys. 99, 498 (2006).

273. Z.X. Wang, S.W. Ding and M.H. Zhang, Chinese J. Inorg. Chem. 21, 437 (2005).

274. Y.C. Lee and S. Cheng, J. Chinese Chem. Soc. 53, 1355 (2006).

275. H. Mattoussi, J.M. Mauro, E.R. Goldman, G.P. Anderson, V.C. Sundar, F.V. Mikulec and M.G. Bawendi, J. Am. Chem. Soc. 122, 12142 (2000).

276. W.C.W. Chan and S.M. Nie, Science 281, 2016 (1998). 
277. M. Bruchez, M. Moronne, P. Gin, S. Weiss and A.P. Alivisatos, Science 281, 2013 (1998).

278. N. Gaponik, D.V. Talapin, A.L. Rogach, K. Hoppe, E.V. Shevchenko, A. Kornowski, A. Eychmuller and H. Weller, J. Phys. Chem. B 106, 7177 (2002).

279. B.O. Dabbousi, J. RodriguezViejo, F.V. Mikulec, J.R. Heine, H. Mattoussi, R. Ober, K.F. Jensen and M.G. Bawendi, J. Phys. Chem. B 101, 9463 (1997).

280. E.R. Goldman, E.D. Balighian, M.K. Kuno, S. Labrenz, P.T. Tran, G.P. Anderson, J.M. Mauro and H. Mattoussi, Phys. Status Solidi B 229, 407 (2002).

281. S. Kim, Y.T. Lim, E.G. Soltesz, A.M. De Grand, J. Lee, A. Nakayama, J.A. Parker, T. Mihaljevic, R.G. Laurence, D.M. Dor, L.H. Cohn, M.G. Bawendi and J.V. Frangioni, Nat. Biotechnol. 22, 93 (2004).

282. E.R. Goldman, A.R. Clapp, G.P. Anderson, H.T. Uyeda, J.M. Mauro, I.L. Medintz and H. Mattoussi, Analyt. Chem. 76, 684 (2004).

283. I.L. Medintz, A.R. Clapp, F.M. Brunel, T. Tiefenbrunn, H.T. Uyeda, E.L. Chang, J.R. Deschamps, P.E. Dawson and H. Mattoussi, Nat. Mater. 5, 581 (2006).

284. A. Agrawal, C.Y. Zhang, T. Byassee, R.A. Tripp and S.M. Nie, Analyt. Chem. 78, 1061 (2006).

285. M.H. Huang, S. Mao, H. Feick, H.Q. Yan, Y.Y. Wu, H. Kind, E. Weber, R. Russo and P.D. Yang, Science 292, 1897 (2001).

286. V.I. Klimov, S.A. Ivanov, J. Nanda, M. Achermann, I. Bezel, J.A. McGuire and A. Piryatinski, Nature 447, 441 (2007).

287. R. Mirin, A. Gossard and J. Bowers, Electronics Letters 32, 1732 (1996).

288. S. Fafard, K. Hinzer, A.J. Springthorpe, Y. Feng, J. McCaffrey, S. Charbonneau and E.M. Griswold, Mat. Sci. Eng. B-Solid 51, 114 (1998).

289. K. Hinzer, C.N. Allen, J. Lapointe, D. Picard, Z.R. Wasilewski, S. Fafard and A.J.S. Thorpe, J. Vac. Sci. Technol. A 18, 578 (2000).

290. K. Hinzer, J. Lapointe, Y. Feng, A. Delage, S. Fafard, A.J. SpringThorpe and E.M. Griswold, J. Appl. Phys. 87, 1496 (2000).

291. S. Tanaka, H. Hirayama, Y. Aoyagi, Y. Narukawa, Y. Kawakami and S. Fujita, Appl. Phys. Lett. 71, 1299 (1997).

292. M.K. Zundel, K. Eberl, N.Y. Jin-Phillipp, F. Phillipp, T. Riedl, E. Fehrenbacher and A. Hangleiter, J. Cryst. Growth. 202, 1121 (1999).

293. V.I. Klimov, A.A. Mikhailovsky, S. Xu, A. Malko, J.A. Hollingsworth, C.A. Leatherdale, H.J. Eisler and M.G. Bawendi, Science 290, 314 (2000).

294. F. Hide, B.J. Schwartz, M.A. Diazgarcia and A.J. Heeger, Chem. Phys. Lett. 256, 424 (1996).

295. S.A. Carter, J.C. Scott and P.J. Brock, Appl. Phys. Lett. 71, 1145 (1997). 
296. T.S. Ahmadi, Z.L. Wang, T.C. Green, A. Henglein, and M.A. ElSayed, Science, 1924 (1996).

297. V.L. Colvin, M.C. Schlamp and A.P. Alivisatos, Nature 370, 354 (1994).

298. N.C. Greenham, X.G. Peng and A.P. Alivisatos, Phys. Rev. B 54, 17628 (1996).

299. S. Nakamura, K. Kitamura, H. Umeya, A. Jia, M. Kobayashi, A. Yoshikawa, M. Shimotomai and K. Takahashi, Electronics Letters 34, 2435 (1998).

300. C.A. Leatherdale, C.R. Kagan, N.Y. Morgan, S.A. Empedocles, M.A. Kastner and M.G. Bawendi, Phys. Rev. B 62, 2669 (2000).

301. H. Mattoussi, A.W. Cumming, C.B. Murray, M.G. Bawendi and R. Ober, Phys. Rev. B 58, 7850 (1998).

302. L. Brus, J. Phys. Chem. 98, 3575 (1994).

303. Z.L. Wang, M.B. Mohamed, S. Link and M.A. El-Sayed, Surface Sci. 440, L809 (1999).

304. W.U. Huynh, X.G. Peng and A.P. Alivisatos, Advan. Mater. 11, 923 (1999).

305. Z.W. Pan, Z.R. Dai and Z.L. Wang, Science 291, 1947 (2001).

306. Y.A. Ono, Annual Review of Materials Science 27, 283 (1997).

307. S. Tanaka, H. Kobayashi and H. Sasakura, in Phosphor Handbook, S. Shionoya and W.M. Yen, Editors, CRC Press, New York, 1999.

308. K. Manzoor, S.R. Vadera, N. Kumar and T.R.N. Kutty, Appl. Phys. Lett. 84, 284 (2004).

309. M. Warkentin, F. Bridges, S.A. Carter and M. Anderson, Phys. Rev. B 75, 075301 (2007).

310. K. Manzoor, V. Aditya, S.R. Vadera, N. Kumar and T.R.N. Kutty, Solid State Commun. 135, 16 (2005).

311. D.J. Norris and M.G. Bawendi, J. Chem. Phys. 103, 5260 (1995).

312. D.J. Norris and M.G. Bawendi, Phys. Rev. B 53, 16338 (1996).

313. A.P. Alivisatos, A.L. Harris, N.J. Levinos, M.L. Steigerwald and L.E. Brus, J. Chem. Phys. 89, 4001 (1988).

314. D.M. Mittleman, R.W. Schoenlein, J.J. Shiang, V.L. Colvin, A.P. Alivisatos and C.V. Shank, Phys. Rev. B 49, 14435 (1994).

315. U. Woggon, S. Gaponenko, W. Langbein, A. Uhrig and C. Klingshirn, Phys. Rev. B 47, 3684 (1993).

316. H. Giessen, B. Fluegel, G. Mohs, N. Peyghambarian, J.R. Sprague, O.I. Micic and A.J. Nozik, Appl. Phys. Lett. 68, 304 (1996).

317. V. Jungnickel and F. Henneberger, J. Luminescence 70, 238 (1996).

318. W.E. Moerner, Science 265, 46 (1994).

319. T. Basche, W.E. Moerner, M. Orrit and U.P. Wild, eds. SingleMolecule Optical Detection, Imaging and Spectroscopy. VCH, Weinheim, Cambridge, 1997.

320. J. Tittel, W. Gohde, F. Koberling, T. Basche, A. Kornowski, H. Weller and A. Eychmuller, J. Phys. Chem. B 101, 3013 (1997). 
321. S.A. Blanton, A. Dehestani, P.C. Lin and P. Guyot-Sionnest, Chem. Phys. Lett. 229, 317 (1994).

322. S.A. Empedocles, D.J. Norris and M.G. Bawendi, Phys. Rev. Lett. 77, 3873 (1996).

323. M. Nirmal, B.O. Dabbousi, M.G. Bawendi, J.J. Macklin, J.K. Trautman, T.D. Harris and L.E. Brus, Nature 383, 802 (1996).

324. S.A. Blanton, M.A. Hines and P. Guyot-Sionnest, Appl. Phys. Lett. 69, 3905 (1996).

325. S.A. Empedocles and M.G. Bawendi, Science 278, 2114 (1997).

326. S.A. Empedocles, R. Neuhauser, K. Shimizu and M.G. Bawendi, Advan. Mater. 11, 1243 (1999).

327. S.A. Empedocles and M.G. Bawendi, J. Phys. Chem. B 103, 1826 (1999).

328. S. Empedocles and M. Bawendi, Acc. chem. Res. 32, 389 (1999).

329. U. Banin, M. Bruchez, A.P. Alivisatos, T. Ha, S. Weiss and D.S. Chemla, J. Chem. Phys. 110, 1195 (1999).

330. T. Matsumoto, M. Ohtsu, K. Matsuda, T. Saiki, H. Saito and K. Nishi, Appl. Phys. Lett. 75, 3246 (1999).

331. N. Murase, Chem. Phys. Lett. 368, 76 (2003). 